P. FORGACS

Z. HORVATH

L. PALLA

\title{
SOLITON THEORETIC FRAMEWORK \\ FOR GENERATING MULTIMONOPOLES
}

\section{Hungarian Academy of Sciences} CENTRAL RESEARCH INSTITUTE FOR PHYSICS 


\title{
SOLITON THEORETIC FRAMEWORK \\ FOR GENERATING MULTIMONOPOLES
}

\author{
P. Forgács \\ Central Research Institute for Physics \\ H-1525 Budapest 114, P.O.B.49, Hungary \\ and \\ Z. Horváth and L. Palla \\ Institute for Theoretical Physics \\ Roland Eötvös University \\ H-1088 Budapest, Hungary
}




\title{
ABSTRACT
}

We present a systematic method using Bäcklund transformation for generating SU(2) Yang-Mills-Higgs monopoles of arbitrary charge. The purely algebraic iteration formula for our Bäcklund transformation is derived. Our method is based on the equivalence of the axially and mirror symmetric Bogomolny equations and the Ernst equation. The properties of the Ernst equation that are relevant for monopoles are also discussed.

The application of the method is illustrated on the example of the one and two monopole solutions.

\begin{abstract}
АННОТАЦИЯ
Предлагается систематический метод для генерации SU(2) монополей произвольного заряда типа Янга-Милльса-Хиггса, основанный на преобразовании Бэклунда. Получена чисто алгебраическая итерационная формула для нашего преобразованкі Бэклунда. Наш метод основан на эквивалентности аксиально и зеркально симметричного уравнения Богомольного и уравенения Эрнста. Обсуждаются те свойства уравнения Эрнста, которые существенны для монополей.

Применение метода иллюстрируется на примере решений с одним и двумя монополями .
\end{abstract}

\section{KIVONAT}

Az SU(2) Yang-Mills-Higgs elmélet véges energiáju, tetszôleges topológikus töltéssel rendelkezô monopólus megoldásainak generálására szolgáló módszert fejlesztünk ki Bäcklund transzformációk fölhasználásával. Levezetünk a Bäcklund transzformációk iterálására vonatkozó, tisztán algebrai formulát. Módszerünk az axiálisan szimmetrikus Bogomolny és az Ernst egyenlet ekvivalenciáján alapul. Az Ernst egyenlet a monopólus elmélet szempontjából fontos tulajdonságait is diszkutáljuk.

A módszer alkalmazásaként megmutatjuk, hogyan lehet generálni az egyszeres töltésü és egy uj, kétszeres töltésü monopólus megoldást. 


\section{INTRODUCTION}

As it was shown for the first time by 'tHooft and Polyakov [I] spontaneously broken gauge theories with a simple gauge group possess classical solutions which may be identified as magnetic monopoles. These solutions can be interpreted as "solitons" in 3+1 dimensions in the sense that their energy is well localised at any instant of time, so they can represent "particles". These particles are stabilized by a quantum number, topological in origin which corresponds to the magnetic charge. Magnetic monopoles arise naturally in the currently popular grand unified theories unifying the weak, electromagnetic and strong interactions. In Ref. [2] it was suggested that particles with both magnetic and electric charges / dyons / may play an important role at the subconstituent level.

In the limit of vanishing Higgs potential, when the Higgs field becomes massless, a considerable simplification arises and the analytic form of the static singly charged and spherically symmetric'tHooft-PoIyakov monopole was found by Prasad and

Sommerfield [3] and by Bogomolny [4]. In this case the theory is considerably simplified and the static minimal energy configurations are solutions of a first order system of equations / Bogomolny equations /. This paper is devoted to the study of these equations. A remarkable property of the theory with vanishing potential that like monopoles do not interact. The repulsive Coulombic force is cancelled by the long range attractive force due to the massless scalar field. 
It has been shown by perturbative techniques $[5,6]$ that the force between monopoles decreases faster than any inverse power of the separation.

The existence of static, noninteracting finite energy monopoles has been recently established by Taubes [7]. The first exact solution corresponding to a doubly charged solution / two monopoles superimposed on each other / was found by Ward [8] using twistor methods and by the present authors [9] . We applied soliton theoretic techniques to generate the one monopole / IMP / [10] and the doubly charged monopole / 2MP / from a simple "vacuum" solution. These results are based on the equivalence of the axially and mirror symmetric Bogomolny equations with a rather well known equation of general relativity, the Ernst equation. [II] . For the Ernst equation there are various solution generating techniques: Bäcklund transformations /BT/ found by Harrison [12] and by Neugebauer [13] and the inverse scattering method of Belinsky and Zakharov [14].

The purpose of this paper is to develop a systematic method outlined in Ref. $[9,10]$ to generate axially symmetric multimonopoles of arbitrary charge. Our procedure is closely related with the inverse scattering methods applied in certain two dimensional systems which are completely integrable.

We introduce a Bäcklund transformation/șlightly different from Harrison's BT / to obtain the monopole solutions. The major advantage of our method that it consists of entirely algebraic steps, and acts directly on the components of vector potential. We have to solve a Riccati type equation only once 
which is easily done for our simple "vacuum" solution. We give in this article a very neat form for the result of iterating an arbitrary number of BT's. We show that by each step the topological charge is increased by one unit.

Our paper is organized as follows: In Section 2. we summarize our notations and discuss the axially and mirror symmetric Bogomolny equations. In Sect. 3. we discuss the relevant properties of the Ernst equation. In Sect. 4. we describe the relationship between the Bogomolny and the self-duality equations. In Sect.6. we display the general formulae for an arbitrary number of BT's. In Sect. 7. we give some special applications.

\section{THE AXIALLY SYMMETRIC EQUATIONS}

We consider an SU (2) gauge theory with an isotriplet Higgs field in the limit of vanishing Higgs potential. The Lagrangian density is

$$
\mathcal{L}=-\frac{1}{4} F_{\mu \nu}^{a} F^{a \mu \nu}-\frac{1}{2}\left(D_{\mu} \Phi\right)^{a}\left(D^{\mu} \Phi\right)^{a}
$$

where $\quad F_{\mu \nu}^{a}=\partial_{\mu} A_{\nu}^{a}-\partial_{\nu} A_{\mu}^{a}-\varepsilon^{a b c} A_{\mu}^{b} A_{\nu}^{c}$

$$
\left(D_{\mu} \Phi\right)^{a}=\partial_{\mu} \Phi^{a}-\varepsilon^{a b c} A_{\mu}^{b} \Phi^{c}
$$

/ We chose the coupling constant $e=1 /$.

The Hamiltonian density for static configurations with no electric fields $/ A_{0}^{a}=0 \quad /$ is 


$$
\begin{aligned}
& \mathcal{H}=\frac{1}{4} F_{i j}^{a} F^{a i j}+\frac{1}{2}\left(D_{i} \Phi\right)^{a}\left(D^{i} \Phi\right)^{a} \\
& (a, i, j=1,2,3)
\end{aligned}
$$

The field equations of this theory are solved by configurations satisfying the Bogomolny equations [4] :

$$
F_{i j}^{a}=-\varepsilon_{i j k}\left(D_{k} \Phi\right)^{a}
$$

The energy, E, can be written using the Bogomolny equations as

$$
E=\int \mathcal{H} d^{3} x=\int\left(D_{k} \Phi\right)^{a}\left(D^{k} \Phi\right)^{a} d^{3} x
$$

Now

$$
\left(D_{k} \Phi\right)^{d}\left(D^{k} \Phi\right)^{a}=\frac{1}{2} \partial^{k} \partial_{k} \Phi^{a} \Phi^{a}=\frac{1}{2} \Delta|\Phi|^{2}
$$

using the equations of motion for the Higgs field, $D^{k} D_{k} \Phi=0$, so the energy can be calculated from the Higgs field alone

$$
E=\frac{1}{2} \int \Delta|\Phi|^{2} d^{3} x
$$

The topological charge, $n$, is given by

$$
n=\lim _{r \rightarrow \infty} \frac{1}{8 \pi v} \int_{r=\text { const. }} d S^{i} \partial_{i}|\Phi|^{2}
$$

Since the asymptotic boundary condition $\lim _{v \rightarrow \infty}|\Phi|=v \quad$ is imposed, for a solution with topological charge $n$ 


$$
|\Phi| \rightarrow v-\frac{n}{r}+\theta\left(\frac{1}{x^{2}}\right) \text { as } x \rightarrow \infty
$$

However, condition ( 8 ) alone does not guarantee that the topological charge is indeed $n$, due to the presence of possible singularities. From now on, we take the vacuum expectation value of the Higgs field $v=1$.

When one is looking for exact solutions of complicated equations, there is a need for a simplifying ansatz consistent with the known properties of the configuration in quest. In the present case the simplest ansatz would be a spherically symmetric one. However, the assumption of spherical symmetry is too strong, it excludes all but the singly charged 'tHooft-Polyakov monopole [15]. The next simplest thing one can do is to assume axial symmetry. In gauge theories by a symmetry we mean that the change of the gauge field, $A_{\mu}^{a}$, under this symmetry transformation / e.g. a rotation around any axis / can be compensated by a gauge transformation $[16]$.

Manton [17] constructed an axially and mirror symmetric ansatz which can be written in polar coordinates as

$$
\begin{aligned}
& A_{0}^{a}=0, \quad \Phi^{a}=\left(0, \phi_{1}, \phi_{2}\right), \quad A_{\varphi}^{a}=-\left(0, \eta_{1}, \eta_{2}\right) \\
& A_{z}^{a}=-\left(w_{1}, 0,0\right), A_{s}^{a}=-\left(w_{2}, 0,0\right)
\end{aligned}
$$

where $x_{1}=\rho \cos \varphi, x_{2}=\rho \sin \varphi$, and $\phi_{i}, \eta_{i}, W_{i}$ functions of $\rho, z$ only. Plugging (10) into the Bogomolny equations (3) they simplify to 


$$
\begin{aligned}
& \partial_{\rho} \phi_{1}-W_{2} \phi_{2}=-\rho^{-1}\left(\partial_{z} \eta_{1}-W_{1} \eta_{2}\right) \\
& \partial_{\rho} \phi_{2}+W_{2} \phi_{1}=-\rho^{-1}\left(\partial_{z} \eta_{2}+W_{1} \eta_{1}\right) \\
& \partial_{\rho} W_{1}-\partial_{z} W_{2}=\rho^{-1}\left(\phi_{1} \eta_{2}-\phi_{2} \eta_{1}\right) \\
& \partial_{z} \phi_{1}-W_{1} \phi_{2}=\rho^{-1}\left(\partial_{s} \eta_{1}-W_{2} \eta_{2}\right) \\
& \partial_{z} \phi_{2}+W_{1} \phi_{1}=\rho^{-1}\left(\partial_{\rho} \eta_{2}+W_{2} \eta_{1}\right)
\end{aligned}
$$

We have five equations for the six unknown variables, however, equations (Ila-e) still possess a residual $U(I)$ gauge invariance: $W_{i}^{\prime}=W_{i}+\partial_{i} \wedge$

$$
\left(\begin{array}{c}
\phi_{i}^{\prime} \\
\eta_{i}^{\prime}
\end{array}\right)=\left(\begin{array}{c}
\phi_{i} \\
\eta_{i}
\end{array}\right) \cos \Lambda+\varepsilon_{i j}\left(\begin{array}{c}
\phi_{j} \\
\eta_{j}
\end{array}\right) \sin \Lambda, \quad \varepsilon_{12}=1=-\varepsilon_{21}
$$

This residual gauge freedom enables us to reduce the number of unknown functions from six to five. In fact, we can do even more than that, namely, we can satisfy one equation in (II) by the following trick: it is possible to find such a $\Lambda$ that

$$
\begin{aligned}
& W_{1}^{\prime}=-\phi_{1}^{\prime} \\
& W_{2}^{\prime}=\frac{1}{\rho} \eta_{1}^{\prime}
\end{aligned}
$$

are simultaneously true. This is more than a gauge fixing, because

(13a) and (13b) imply equations (11a-c) for $\phi_{i}, \eta_{i}, W_{i}$. To show this explicitly, one should derive the integrability conditions for equations $(13 a-b)$, which are easily found to be

$$
\begin{aligned}
& \partial_{z} W_{2}-\partial_{s} W_{1}=\rho^{-1}\left(\eta_{1} \phi_{2}-\eta_{2} \phi_{1}\right)+\sin \Lambda\left(\phi_{2, \rho}+\phi_{1} W_{2}+\right. \\
& \left.+\rho^{-1}\left[\eta_{2, z}+\eta_{1} W_{1}\right]\right)+\cos \Lambda\left(\phi_{1, s}-W_{2} \phi_{2}+\rho^{-1}\left[\eta_{1, z}-\eta_{2} W_{1}\right]\right)
\end{aligned}
$$


Eq. (14) really implies (11a-c), so, for a solution of (11a-c) it is possible to require both conditions $(13 a-b)$ at once. Now, using $(13 a-b)$ eqs. (11a-e) reduce to four equations for four unknown functions. Next, we observe that (11b) implies the existence of a function $f$, such that

$$
\phi_{2}=-\frac{f, z}{f} ; \quad \frac{1}{s} \eta_{2}=\frac{f_{, s}}{f}
$$

Then it is easy to see that (11c) can be also satisfied by putting

$$
W_{1}=-\frac{\Psi_{\text {,z }}}{f}, W_{2}=-\frac{\Psi_{\text {, }}}{f}
$$

and the remaining two equations (11d-e) reduce to

$$
\begin{aligned}
& f \Delta f-(\nabla f)^{2}+(\nabla \psi)^{2}=0 \\
& f \Delta \psi-2 \nabla f \nabla \psi=0
\end{aligned}
$$

where $\Delta=\partial_{s}^{2}+s^{-1} \partial_{s}+\partial_{z}^{2} \quad, \nabla=\left(\partial_{z}, \partial_{s}\right)$ Introducing $\epsilon=f+i \psi$ eqs. (17a-b) may be written as

$$
\operatorname{Re} \epsilon \Delta \epsilon-(\nabla \epsilon)^{2}=0
$$

which is the celebrated form of the Ernst equation of general relativity. A geometrical derivation of eq. (18) from (1la-e) was given in Ref. [10].

The equivalence of eqs. (1la-e) and (18) is of interest because it reveals a surprising / although completely 
formal / connection between general relativity and SU(2) gauge theories, and what is more important for us, it gave the clue to find new exact solutions of the Bogomolny equations describing multiply charged monopoles, using the various solution generating techniques worked out for the Ernst equation.

As we would like to make this paper somewhat self-contained, in the next section we summarize briefly those properties of this equation that are relevant in the study of monopoles. For a more detailed discussion of this important equation in general relativity we refer to the literature $[18,19]$.

\section{PROPERTIES OF THE ERNST EQUATION}

The most general line element of an axially symmetric, stationary space-time can be written as

$$
d s^{2}=f(d t-\omega d \varphi)^{2}-\frac{1}{f}\left[e^{2 \gamma}\left(d \rho^{2}+d z^{2}\right)+\rho^{2} d \varphi^{2}\right]
$$

where $f, w, \gamma \quad$ are functions of $\rho, z$ only. The vacuum Einstein equations corresponding to (19) are

$$
\begin{aligned}
& \Delta \ln f+\frac{f^{2}}{s^{2}}(\nabla \omega)^{2}=0 \\
& \frac{\rho}{f} \Delta \omega-2 \nabla \omega \nabla \frac{s}{f}=0 \\
& -\Delta 2 \gamma=\frac{f^{2} z}{f^{2}}+\frac{f^{2}}{\rho^{2}} \omega_{, s}^{2}
\end{aligned}
$$




$$
\begin{aligned}
& 2 \gamma_{, z}=\rho \frac{f_{\partial \rho} f_{j z}}{f^{2}}-\frac{f^{2}}{\rho} \omega_{\partial \rho} \omega_{, z} \\
& 2 \gamma_{\partial \rho}=\frac{\rho}{2} \frac{f_{\partial \rho}^{2}-f_{, z}^{2}}{f^{2}}-\frac{f^{2}}{2 \rho}\left(\omega_{, \rho}^{2}-\omega_{\partial z}^{2}\right)
\end{aligned}
$$

Once eqs. $(20 a, b)$ are solved, it is easy to integrate eqs. $(21 a-c)$ for $\gamma$, so we shall concentrate on eqs. $(20 a, b)$. Eqs. (20a,b) possess an SL $(2, R)$ symmetry group which corresponds to the linear coordinate transformations:

$$
\begin{aligned}
& t=d t^{\prime}-b \varphi^{\prime} \\
& \varphi=a \varphi^{\prime}-c t^{\prime} \quad(a d-b c=1)
\end{aligned}
$$

This SL $(2, R)$ invariance group will be denoted by L. Rewriting eqs. $(20 a, b)$ as

$$
\begin{aligned}
& \frac{\eta_{+}+\eta_{-}}{2} \Delta \eta_{+}-\left(\nabla \eta_{+}\right)^{2}=0 \\
& \frac{\eta_{+}+\eta_{-}}{2} \Delta \eta_{-}-\left(\nabla \eta_{-}\right)^{2}=0
\end{aligned}
$$

where $\eta_{ \pm}=\frac{s}{f} \pm w$ - $\eta_{ \pm}$transforms under the action of $\mathrm{L}$ as

$$
\eta_{ \pm}^{\prime}=\frac{a \eta_{ \pm} \pm b}{d \pm c \eta_{ \pm}}
$$

Now we observe that eq. (20b) can be interpreted as the integrability condition for the existence of a new function $\psi$ which is related to $\omega$ in the following way: 


$$
\psi_{\partial \rho}=\frac{f^{2}}{\rho} \omega_{, z} ; \quad \psi_{\partial z}=-\frac{f^{2}}{\rho} \omega_{>s}
$$

Introducing the complex Ernst potential, $\epsilon=f+i \psi$, eqs. $(20 a, b)$ take the form: $\operatorname{Re} \in \Delta \epsilon-(\nabla \epsilon)^{2}=0$ which is just eq, (18). The Ernst eq. (18) is invariant under a new $\mathrm{SL}(2, \mathrm{R})$ transformation group, P , / Ehlers transformations [20] / defined by

$$
\epsilon^{\prime}=\frac{\alpha \epsilon+i \beta}{\delta-i \gamma \epsilon}, \quad \alpha \delta-\beta \delta=1
$$

It is important to realize that there exists a direct mapping from eqs. (18) to eqs. (20a,b) / this is the so called NeugebauerKramer mapping [2I] /. This mapping is a group element, $I=I^{-1}$, of a cyclic group of order two, defined by

$$
f^{\prime}=\frac{\rho}{f}, \quad \omega^{\prime}=-i \psi, \quad \psi^{\prime}=i \omega
$$

We shall write (27) in a more compact form

$$
I\{f, \omega, \psi\}=\left\{\frac{s}{f},-i \psi, i \omega\right\}
$$

In fact, the groups $L$ and $P$ are related by $I$ :

$$
I I I^{-1}=P \text {. }
$$

The action of the group of coordinate transformations, L, does not commute with the Ehlers transformations, $P$, and by the repeated applications of these transformations one can generate new solutions of the Ernst equation from the old ones. However, 
properties of solutions generated this way change in an uncontrollable way, e.g. starting with an asymptotically flat solution the result will be an asymptotically non-flat space-time which is of little use in general relativity. In fact, we have an infinite dimensional symmetry group - the Geroch group [22] - of the Ernst equation and one should look for suitable subgroups which preserve asymptotic flatness to generate physically interesting solutions in general relativity. This program was completed by Kinnersley et al [23] - Geroch has shown the existence of infinitely many conservation laws, as well. The origin of the Geroch group was somewhat mysterious until the discovery that it can be related to the existence of a Lax pair [24] . A method was developped by Belinski and Zakharov [14] to solve the resulting linear eigenvalue problem. Independently of each other Harrison [12] and Neugebauer [13] found Bäcklund transformations / BT / for the Ernst equation. Neugebauer deduced a composition theorem for two successive BT's, so once a BT has been determined one can apply arbitrary many BT's by algebraic methods alone. Cosgrove [19] has found a composition theorem for Harrison's BT's using Neugebauer's work. All these techniques could be successfully applied to generate solutions of physical relevance / asymptotically flat / for the Ernst equation containing arbitrary many free parameters. In general relativity for most solutions of interest, the Ernst potential, $\epsilon$, can be written as

$$
\epsilon=1-\frac{2 m}{r}+\frac{1}{r} \sum_{n} A_{n}(\vartheta) r^{-n}
$$


$/ \gamma_{1} \vartheta$ are the usual spherical coordinates, $m$ is the / real / Schwarzshild mass /. However, if one is interested in generating solutions of the Ernst equation corresponding to magnetic monopoles, one needs a completely different condition of (29) - The gauge invariant length of the Higgs field is given by

$$
\phi^{2}=\phi_{1}^{2}+\phi_{2}^{2}=\frac{f_{, z}^{2}+\psi_{, z}^{2}}{f^{2}}
$$

It is important to note here that the Ehlers transformations (26) leave $\phi^{2}$ invariant, whereas the $I$ and $L$ transformations change $\phi^{2} \quad$ - Our boundary condition requires that for a monopole of charge $n$,

$$
\phi^{2} \rightarrow 1-\frac{2 n}{r}+\theta\left(\frac{1}{r^{2}}\right) \text { as } r \rightarrow \infty
$$

which is just incompatible with eq. (29). The moral from this is that unphysical solutions in general relativity might be of interest in gauge theories and vice versa.

We think, it is clear from the above that one should be somewhat careful in applying the techniques working so beatifully in general relativity for generating multimonopole solutions due to the fact that the boundary conditions are so different. In fact, it is a very intrigueing feature of the Ernst equation that both the Kerr and the monopole solution can be interpreted as "solitons" in the sense that both can be generated by BT's starting with the "vacuum" of general relativity / Minkowsky space / or with the Higgs vacuum $/ \phi^{2}=1 \quad /$ respectively. 
Before entering into the details of explaining how Bäcklund transformations can be used to generate multimonopole solutions, we make a short digression to show the connection between the Ernst equation and its symmetry transformations on the one hand and the selfduality equations and the non-linear transformations of Corrigan et al [26] on the other hand.

4. THE CONNECTION BETWEEN NONOPOLES AND SELFEDUAL GAUGE FIELDS

Let us consider a pure SU (2) gauge theory with Lagrangian

$$
\mathcal{L}=-\frac{1}{4} F_{\mu \nu}^{a} F^{a \mu r}
$$

The Euclidean space field equations are solved by such configurations which satisfy the self-duality conditions

$$
F_{\mu \nu}^{a}={ }^{*} F_{\mu \nu}^{a}
$$

where ${ }^{*} F_{\mu_{\gamma}}^{a}$ is the dual field tensor

$$
{ }^{*} F_{\mu v}^{a}=\frac{1}{2} \epsilon_{\mu v s \sigma} F^{a s \sigma}
$$

In the case when all fields, $A_{\mu}^{a}$, are independent of the Euclidean time the self-duality equations (33) reduce to

$$
F_{i j}^{a}=-\varepsilon_{i j k}\left(D_{k} A_{0}\right)^{a}
$$


where we recognize the Bogomolny eqations (3), reinterpreting $A_{0}^{a}$ as the Higgs field.

Yang has shown the existence of a particularly convenient gauge $\rightarrow$ the R-gauge - in which the self-duality equations take the following form [25] :

$$
\begin{aligned}
& x\left(x_{, y \bar{y}}+\chi_{, z \bar{z}}\right)-x_{\partial y} x_{, \bar{y}}-x_{, z} x_{, \bar{z}}+\tau_{, y} \bar{\tau}_{, \bar{y}}+\tau_{, z} \bar{\tau}_{, \bar{z}}=0 \\
& x\left(\tau_{, y \bar{y}}+\tau_{, z \bar{z}}\right)-2 \tau_{\partial y} x_{, \bar{y}}-2 \tau_{, z} x_{, \bar{z}}=0 \\
& x\left(\bar{\tau}_{, y \bar{y}}+\bar{\tau}_{\partial \bar{z}}\right)-2 \bar{\tau}_{, \bar{y}} x_{, y}-2 \bar{\tau}_{, \bar{z}} x_{, z}=0
\end{aligned}
$$

$$
\sqrt{2} y=x_{1}+i x_{2}, \sqrt{2} z=x_{3}+i x_{4}
$$

It is useful to introduce a new function $\pi$ realising that $(36 \mathrm{~b}, \mathrm{c})$ are identically satisfied defining $\pi$ as

$$
-\chi^{-2} \tau_{j z}=\bar{\pi}, \bar{y} \quad ; \quad \chi^{-2} \tau_{, y}=\bar{\pi}_{, \bar{z}}
$$

$/$ and similarly for the barred quantities /. In terms of $\chi, \pi$, $\bar{\pi}$ the self-duality equations take the form

$$
\begin{aligned}
& \chi\left(\chi_{\partial y \bar{y}}+\chi_{\partial z \bar{z}}\right)-\chi_{j y} \chi_{, \bar{y}}-\chi_{j z} \chi_{j \bar{z}}+\chi^{4}\left(\pi_{j y} \bar{\pi}_{\partial \bar{y}}+\pi_{j z} \bar{\pi}_{, \bar{z}}\right)=0 \\
& \left(\chi^{2} \bar{\pi}_{, \bar{y}}\right)_{, y}+\left(\chi^{2} \bar{\pi}_{\bar{z}}\right)_{, z}=0 \\
& \left(\chi^{2} \pi, y\right)_{\bar{y}}+\left(\chi^{2} \pi_{, z}\right)_{\bar{z}}=0
\end{aligned}
$$

The striking similarity of eqs. $(36 \mathrm{a}-\mathrm{c})$ to the Ernst equations $(17 a, b)$ and of $(36 d-f)$ to $(20 a, b)$ makes it obvious how to relate the former systems of equations to the latter ones. Indeed, taking $\tau$ to be real and assuming that both $\chi$ and $\tau$ depend only on $\xi, x_{3}$ we immediately obtain eqs. (17a,b) [27]. However, taking $X=s f\left(s, x_{3}\right), \tau=\sqrt{2} \bar{y} \psi\left(s, x_{3}\right)$ 
$\pi=\left(2 \bar{y}^{2}\right)^{-1} w\left(s, x_{3}\right)$ in eqs. (36)/ with $f, \psi$ and $w$ being real functions / we again obtain $(17 a, b)$ and $(20 a, b)$ respectively. This is important as it is also possible to show that using these latter identifications for $\chi, \tau, \pi$ we obtain the expressions $(13,15,16)$ for the $\phi_{i}, \eta_{i}, W_{i}$ functions of the Manton ansatz.

As it was pointed out by Corrigan et al. [26] the selfduality equations $(36 a-c)$ have an $\mathrm{SL}(2, \mathrm{C})$ invariance group which acts nonlinearly on $\chi$ and $\tau$ :

$$
\begin{aligned}
x \rightarrow \frac{x}{(a+b \tau)(\bar{a}+\bar{b} \bar{\tau})+b \bar{b} \chi^{2}} \\
\tau \rightarrow \frac{(c+d \tau)(\bar{a}+\bar{b} \tau)+\bar{b} d \chi^{2}}{(a+b \tau)(\bar{a}+\bar{b} \tau)+b \bar{b} \chi^{2}}
\end{aligned}
$$

This transformation was denoted by $\gamma$ in Ref. [26]. It is easy to see that $(37 a, b)$ are the analogues of the Ehlers transformations for the Ernst equation discussed in Sect.3. It is important to realize that in the $S L(2, c)$ transformation group (37) a,b,c,d can be taken not just simple parameters but arbitrary analytical functions of $(y, z)[28]$. In what follows we shall use a simple subgroup of this SL $(2, C)$ group that maps $(X, \tau, \bar{\tau}, \bar{\pi}, \bar{\pi})$ into $\left(|A| \chi, \bar{A} \tau, A \bar{\tau}, \bar{A}^{-1} \pi, A^{-1} \bar{\pi}\right) \quad$ where $A=A(y)$ is an arbitrary analytic function.

In Ref. [26] yet another invariance transformation / the so called $\beta$ or "Bäcklund" transformation / was also given: $\begin{array}{ll}\text { if }(x, \tau, \bar{\tau}, \pi, \bar{\pi}) & \text { satisfy eqs. (36) then } \\ \left(\chi^{-1}, i \pi, i \bar{\pi},-i \tau,-i \bar{\tau}\right) & \text { are also solutions of these }\end{array}$ 
equations. Note that this is a discrete transformation yielding the identity when applied twice. This fact and the structure of the $\beta$ transformation indicate that $\beta$ can be related to the Neugebauer-Kramer mapping (27) . For example, in the case of the Manton ansatz we find that $\beta$ acts on $f, \psi, \omega$ as follows:

$$
\left(\rho f, \sqrt{2} \bar{y} \psi, \sqrt{2} y \psi, \frac{\omega}{2 \bar{y}^{2}}, \frac{\omega}{2 y^{2}}\right) \stackrel{\beta}{\longrightarrow}\left(\frac{1}{\rho f}, \frac{i \omega}{2 \bar{y}^{2}}, \frac{i \omega}{2 y^{2}},-i \sqrt{2} \bar{y} \psi,-i \sqrt{2} y \psi\right)
$$

This is yet not equivalent to eq. (27), however, applying to it an SL $(2, C)$ transformation of the type we mentioned above with $A(y)=2 \sqrt{2} y^{3} \quad$ we indeed obtain the Neugebauer-Kramer mapping for $f, \Psi, w$. Thus we see the precise connection - within the Manton ansatz - between the $\beta$ transformation of Ref. [26] and the Neugebauer-Kramer mapping eq. (27) : the latter is the product of $a \quad \beta$ and a special SL $(2, C)$ transformation with a given analytic function.

In our opinion it would be more appropriate to make a distinction between Bäcklund transformations and the $\beta$ and $\delta$ transformations of Ref. [26] , the latter being rather an invariance property of the Yang equations. It seems reasonable to expect that BT's for eqs. $(36 \mathrm{a}-\mathrm{c})$ should reduce to those already known for the Ernst equation in a special case, just as the SL (2,C) transformations (37) give the Ehlers transformations when we restrict eqs. $(36 \mathrm{a}-\mathrm{c})$ to the Ernst equation. As far as we know BT's in this sense / generalizing Harrison's or Neugebauer's BT's / are not yet found. There is a strong indication that these BT's really exist since in Ref. [28] the existence of the corresponding linear eigenvalue problem which reduces to 
that of Belinski and Zakharov for the Ernst equation [14] was.. discovered. In Ref. [28] we constructed a four dimensional "soliton" generating method to solve this linear eigenvalue problem which is the generalization of the method of Belinski and Zakharov. On the other hand, it has been proved by Cosgrove [19] that the soliton generating method of Belinski and Zakharov is in close connection with Harrison's BT.

In Ref. [28] it was shown that eqs. (36a-c) possess an infinite parameter invariance group which is the analogue of the Geroch group. In applying this invariance group for generating new solutions for the Yang equations one encounters problems similar to the simpler case of the Ernst equation. A straightforward application does not lead very far because of the uncontrollable singularities. Lohe [29] tried to generate multimonopole solutions of eqs. $(36 a-c)$ when $\chi$ and $\tau$ are independent of $x_{4}$ / which are of course the Bogomolny equations / carrying out this procedure on the BPS monopole. He applied the special product $\beta \delta \beta$ to ensure the reality of the final result which turned out to be a singular configuration. This is not surprising remembering that this method does not lead to acceptable solutions in the case of general relativity if we start from an asymptotically flat solution. To get physically interesting solutions / e.g. those with finite action / one should find suitable subgroups in a spirit similar to the work of Kinnersley et al.

As it was shown by Manton [17] the Bogomolny-PrasadSommerfield /BPS/ monopole $[3,4]$ can be obtained from the Corrigan-Fairlie-'tHooft-Wilczek / CFtHW / ansatz [30] in a 
complex gauge, but he found that the CFtHW ansatz does not contain multimonopole solutions.

Atiyah and Ward [3I] found a natural generalization of the CFtHW ansatz which arose out of the twistor approach for the self-duality equations, originally proposed by Ward [32] . The construction of Atiyah and Ward leads to a hierarchy of ansatze $A_{1}, A_{2}, \ldots$, where $A_{1}$ is just the CFtHW ansatz. Ward used the $A_{2}$ ansatz and found a doubly charged monopole by a somewhat ad hoc generalization of the BPS solution [8] . Independently of Ward we found the same solution using the methods described in this paper [9].

5. BÄCKLUND TRANSFORMATIONS FOR THE ERNST EQUATION

Since for generating multimonopole solutions we found most convenient to apply a slightly modified form of Harrison's $\mathrm{BT} / \mathrm{HBT} /$, and the existing publications on this topic are somewhat compressed, it might be of use to enter into some details. We present the material from a different point of view from that of general relativity. We show how one can derive the composition theorem for HBT's from first principles / without referring to Neugebauer's composition theorem / and derive the general formulae for an arbitrary number of HBT's.

As a first step, we discuss Harrison's Bäcklund transformation and derive the composition theorem for two consecutive 
BT's. This composition theorem is of fundam mal importance since it enables us to construct multisoliton solutions using pure algebra once a Riccati equation for the first step has been solved. We define the following variables:

$$
\begin{array}{ll}
M_{1}=\frac{\epsilon_{11}}{2 f} ; & M_{2}=\frac{\epsilon^{*}, 1}{2 f} ; \quad N_{1}=\frac{\epsilon^{*}, 2}{2 f} ; \\
N_{2}=\frac{\epsilon_{32}}{2 f} ; \quad \epsilon_{21}=\frac{\partial \epsilon}{\partial \zeta_{1}} ; \quad \epsilon_{22}=\frac{\partial \epsilon}{\partial \zeta_{2}} ; \zeta_{1}=\rho+i z ; \zeta_{2}=\rho-i z
\end{array}
$$

These variables are different from that of Harrison, the connection between (38) and that of Ref. [12] was pointed out in Ref. [10]. The Ernst equation (18) can be recast into a system of first order differential equations for the variables (38)

$$
\begin{aligned}
& M_{1,2}=-M_{1} N_{1}+M_{1} N_{2}-\frac{1}{2\left(\zeta_{1}+\zeta_{2}\right)}\left(M_{1}+N_{2}\right), \\
& M_{2,2}=-M_{2} N_{2}+M_{2} N_{1}-\frac{1}{2\left(\zeta_{1}+\zeta_{2}\right)}\left(N_{1}+M_{2}\right), \\
& N_{1,1}=-M_{1} N_{1}+M_{2} N_{1}-\frac{1}{2\left(\zeta_{1}+\zeta_{2}\right)}\left(N_{1}+M_{2}\right), \\
& N_{2,1}=-M_{2} N_{2}+N_{2} M_{1}-\frac{1}{2\left(\zeta_{1}+\zeta_{2}\right)} M_{1}+N_{2},
\end{aligned}
$$

Harrison derived his BT following the method of Wahlquist and Estabrook [33] . He started with rewriting eqs. (39a-d) as four 2-forms which are to vanish for a solution. Then he looked for a pseudopotential $q$ such that the l-form 


$$
\sigma=-d q+F\left(q, N_{i}, M_{i}, \zeta_{i}\right) d \zeta_{I}+G\left(q, N_{i}, M_{i}, \zeta_{i}\right) d \zeta_{2}
$$

satisfies $d \sigma=0$ if eqs. (39) are satisfied and $\sigma=0$. Furthermore, he supposed that $F$ and $G$ depend only linearly on $M_{i}$ 's and $N_{i}$ 's. This assumption guarantees that the resulting equation for the pseudopotential, $q$, will be of Riccati type. Indeed, this way one obtains a total Riccati equation for $q\left(\zeta_{1}, \zeta_{2}\right)$ :

$$
\begin{aligned}
d q= & {\left[\left(M_{2}-M_{1}\right) q+p(w)\left(M_{2}-M_{1} q^{2}\right)\right] d \zeta_{1}+} \\
& +\left[\left(N_{1}-N_{2}\right) q+p^{-1}(w)\left(N_{1}-N_{2} q^{2}\right)\right] d \zeta_{2},
\end{aligned}
$$

where $p(w)=\sqrt{\left(w-i \zeta_{2}\right)\left(w+i \zeta_{1}\right)^{-1}}$, w being a constant of integration. We remark here that the other integration constant for $q$ will be denoted by $\beta$.

In order to find the concrete form of the BT he looked for a new solution of eqs. (39) which depends only on $\zeta_{i}, M_{i}$, $\mathrm{N}_{\mathrm{i}}$, q. This procedure leads to the following form of the BT:

$$
\begin{aligned}
& H(q, p) M_{1}=M_{1}^{(1)}=-q \frac{I+p q}{p+q} M_{1}-\frac{q}{4 \rho} \frac{p^{2}-1}{p+q}, \\
& H(q, p) M_{2}=M_{2}^{(1)}=-\frac{p+q}{q(1+p q)} M_{2}-\frac{1}{4 \rho} \frac{p^{2}-1}{1+p q}, \\
& H(q, p) N_{1}=N_{1}^{(1)}=-\frac{1+p q}{q(p+q)} N_{1}-\frac{1}{4 \rho} \frac{1-p^{2}}{p(p+q)}, \\
& H(q, p) N_{2}=N_{2}^{(1)}=-q \frac{p+q}{1+p q} N_{2}-\frac{q\left(1-p^{2}\right)}{4 \rho p(1+p q)},
\end{aligned}
$$


It will be convenient for us to show at this stage how the Neugebauer-Kramer mapping, I, acts on the $M_{i}$ 's, $N_{i}^{\prime} s$ and on q. A straightforward calculation using the definitions (26) and (27) gives

$$
\begin{aligned}
& I M_{1}=-M_{2}+\frac{I}{4 \rho}, \quad I M_{2}=-M_{1}+\frac{I}{4 \rho}, \\
& I N_{1}=-N_{1}+\frac{I}{4 \rho}, \quad I N_{2}=-N_{2}+\frac{I}{4 \rho},
\end{aligned}
$$

We define the action of $I$ on $q$ in the follwing way: Iq $\bar{q}$ satisfies

$$
\begin{aligned}
d \bar{q}= & {\left[-M_{1}(\bar{q}+p)+M_{2} \bar{q}(1+p \bar{q})+\frac{p}{4 \rho}\left(1-\bar{q}^{2}\right)\right] d \zeta_{1}+} \\
& +\left[-N_{1}\left(\bar{q}+\frac{1}{p}\right)+N_{2} \bar{q}\left(1+\frac{\bar{q}}{p}\right)+\frac{1}{4 p \rho}\left(1-\bar{q}^{2}\right)\right] d \zeta_{2} .
\end{aligned}
$$

Eq. (43) comes from eq. (40) for the pseudopotential, q, replacing there $M_{i}^{\prime} s, N_{i}^{\prime} s$ by $I M_{i}^{\prime} s$ and $I N_{i}$ 's.

It is not difficult to verify that $\bar{q}$ is given in terms of $\mathrm{p}$ and $\mathrm{q} /$ satisfying eq. (40) / as

$$
\bar{q}=-\frac{p+q}{1+p q}
$$

At this stage it seems to be very difficult to apply a second $\mathrm{BT}$, because we have to solve eq. (40) again replacing the $M_{i}^{\prime} s, N_{i}^{\prime} s$ by $M_{i}^{(1)}$ 's and $N_{i}^{(1)}$ 's to find $q$ ' for the next step. 
Usually this is a very hard task even for the simplest seed solutions, since after a single BT the new configurations can be rather complicated. The great advantage of the BT is the existence of a composition theorem stating that there is no need to solve analytically this complicated equation as its solution can be constructed in an appropriate / algebraic / way from solutions of (40) with the original $\mathrm{M}_{i}$ 's and $\mathrm{N}_{i}$ 's.

We now proceed to derive this composition theorem for two consecutive HBT's which will enable us to find the general formulae for an arbitrary number of steps by algebraic methods alone. Let us suppose that eq. (40) for the pseudopotential, $q$, was solved. Making use of (4I) we can perform the first BT. Let us suppose that for the second BT $q^{\prime}$ depends only on $\left(p_{1}, p_{2}\right.$, $\left.q_{1}, q_{2}\right)$ where $\left(p_{1}, q_{1}\right)$ and $\left(p_{2}, q_{2}\right)$ are both solutions of $(40)$ with the same $M_{i}$ 's, $N_{i}$ 's but different constants $w_{1}, w_{2}, \beta_{1}, \beta_{2}$. This assumption is motivated by other work in soliton theory / Sine-Gordon, Korteweg-de Vries, nonlinear Schrödinger equations /. This way we obtain two equations for dq', namely, eq. (40) with $H\left(q_{1}, p_{1}\right) M_{i}$, etc. and the other coming from our assumption for $q^{\prime}$. After equating them as it is shown in detail in Appendix $A$ we obtain an overdetermined system of equations for $q^{\prime}$ which can be consistently solved yielding

$$
q^{\prime}=\frac{\bar{q}_{1} p_{1}-\bar{q}_{2} p_{2}}{q_{1}\left(\bar{q}_{1} p_{1}-\bar{q}_{2} p_{2}\right)}
$$

Writing out (45) in terms of $p_{1}, p_{2}, q_{1}, q_{2}$ we get the formula derived by Cosgrove [19]: 


$$
q^{\prime}=\frac{p_{2}\left(1-p_{1}^{2}\right) q_{1}-p_{1}\left(1-p_{2}^{2}\right) q_{2}+\left(p_{2}^{2}-p_{1}^{2}\right) q_{1} q_{2}}{q_{1}\left[\left(p_{1}^{2}-p_{2}^{2}\right)+p_{1}\left(1-p_{2}^{2}\right) q_{1}-p_{2}\left(1-p_{1}^{2}\right) q_{2}\right]} .
$$

Since we will use the transformations $\operatorname{IH}\left(p_{i}, q_{i}\right)$ to generate the monopoles we now turn our attention to the effect of this transformation on the $M_{i}^{\prime} s$ and $N_{i}$ 's. Henceforth the $\mathrm{IH}\left(p_{i}, q_{i}\right)$ transformation will be denoted by $B\left(p_{i}, q_{i}\right)$. It is easily verified that

$$
\begin{aligned}
& \text { B } M_{1}^{(0)}=-\bar{q}\left(\frac{1}{q} M_{2}^{(0)}+\frac{p}{4 \rho}\right) \text {, } \\
& \text { B } M_{2}^{(0)}=-\frac{1}{\bar{q}}\left(\mathrm{qM}_{1}^{(0)}+\frac{p}{4 s}\right) \text {, } \\
& B N_{1}^{(0)}=-\frac{1}{\bar{q}}\left(\frac{1}{q} N_{1}^{(0)}+\frac{1}{4 p_{g}}\right) \text {, } \\
& B N_{2}^{(0)}=-\bar{q}\left(\mathrm{qN}_{2}^{(0)}+\frac{1}{4 \mathrm{ps}}\right) \text {. }
\end{aligned}
$$

As we shall iterate $B$ we need the following commutation property, of the $\mathrm{H}$ and I transformations which can be derived without difficulty from (4I), (42) :

$$
B(p, q)=I H(p, q)=H(p, \vec{q}) I=I^{-1} B(p, \bar{q}) I
$$

To illustrate the use of (48) we show how one can reduce IHIH. The pseudopotential in the argument of the second $H$ transformation is of course not $q^{\prime}$ but $\bar{q}^{\prime}$, since it is preceded by an I mapping. So 


$$
\text { I H }\left(p_{2}, \bar{q},\right) \text { I H }\left(p_{1}, q_{1}\right)=H\left(p_{2}, q^{\prime}\right) H\left(p_{1}, q_{1}\right)
$$

where we used that $I^{2}=1$.

6. SUPERPOSITION FORMULAE FOR AN ARBITRARY NUMBER OF BÄCKLUND TRANSFORMATIONS

In this section we give the general formulae for $\mathrm{n}$ consecutive B transformations. Since the proof of this result is rather technical we present it in Appendix B.

It is important to deal with the reality conditions for solutions generated by this method. The conditions

$$
M_{1}^{*}=N_{1}, M_{2}^{*}=N_{2}
$$

will obviously ensure the reality of the new solution. For example, after a single $B$ step it immediately follows from (47) that starting with a real solution, $\epsilon_{0}$, for which $\psi_{0}=0$ eq. (50) implies

$$
q=q^{*}, \quad p^{*}=p^{-1}
$$

In a different case one should determine what are the constraints following from (50) for the q's and p's.

It is illuminating to show how to derive the formulae 
for two B steps which was applied in Ref. [9] . We proceed to carry out a second B transformation for (47):

$$
M_{1}^{(2)}=B M_{1}^{(1)}=-q \cdot\left(\frac{1}{\bar{q},} M_{2}^{(1)}+\frac{p_{2}}{4 \rho}\right)
$$

Now applying the duality principle we get for $\bar{q}$ ' / see Appendix A /

$$
\bar{q}=\frac{q_{1} p_{2}-q_{2} p_{1}}{\bar{q}_{1}\left(q_{1} p_{1}-q_{2} p_{2}\right)}
$$

Substituting expressions (45) (47b) and (53) into (52) after some simple algebra we find

$$
M_{1}^{(2)}=\frac{\bar{q}_{1} p_{2}-p_{1} \bar{q}_{2}}{p_{1} \bar{q}_{1}-p_{2} \bar{q}_{2}}\left[\frac{p_{1} q_{1}-p_{2} q_{2}}{p_{2} q_{1}-p_{1} q_{2}} M_{1}^{(0)}+\frac{p_{1}^{2}-p_{2}^{2}}{4 \rho\left(p_{2} q_{1}-p_{1} q_{2}\right)}\right] \cdot(54 a)
$$

Similarly for the other components

$$
\begin{aligned}
& M_{2}^{(2)}=\frac{p_{1} \bar{q}_{1}-p_{2} \bar{q}_{2}}{\bar{q}_{1} p_{2}-p_{1} \bar{q}_{2}}\left[\frac{p_{2} q_{1}-p_{1} q_{2}}{p_{1} q_{1}-p_{2} q_{2}} M_{2}^{(0)}+\frac{q_{1} q_{2}\left(p_{1}^{2}-p_{2}^{2}\right)}{4 \rho\left(p_{2} q_{2}-p_{1} q_{1}\right)}\right],(54 b) \\
& N_{1}^{(2)}=\frac{p_{1} \bar{q}_{1}-p_{2} \bar{q}_{2}}{\bar{q}_{1} p_{2}-p_{1} \bar{q}_{2}}\left[\frac{p_{1} q_{1}-p_{2} q_{2}}{q_{1} p_{2}-p_{1} q_{2}} N_{1}^{(0)}+\frac{q_{1} q_{2}\left(p_{2}^{2}-p_{1}^{2}\right)}{4 \rho p_{1} p_{2}\left(p_{1} q_{2}-p_{2} q_{1}\right)}\right],(54 c) \\
& N_{2}^{(2)}=\frac{\bar{q}_{1} p_{2}-p_{1} \bar{q}_{2}}{p_{1} \bar{q}_{1}-p_{2} \bar{q}_{2}}\left[\frac{p_{2} q_{1}-p_{1} q_{2}}{p_{1} q_{1}-p_{2} q_{2}} N_{2}^{(o)}+\frac{p_{2}^{2}-p_{1}^{2}}{4 \rho p_{1} p_{2}\left(p_{1} q_{1}-p_{2} q_{2}\right)}\right], \text { (54d) }
\end{aligned}
$$


From condition (50) we deduce that either

$$
p_{1}^{*}=p_{2}^{-1}, q_{1}^{*}=q_{2}^{-1}
$$

or

$$
p_{i}^{*}=p_{i}^{-1}, q_{i}^{*}=q_{i}^{-1} \quad(i=1,2)
$$

Eqs. (55) and (56) reduce the number of arbitrary parameters. We now rewrite eqs. (54) using determinants which is very suggestive. It is sufficient to consider $(54 a, d)$ only

$$
\begin{aligned}
& M_{1}^{(2)}=\frac{\left|\begin{array}{ll}
\bar{q}_{1} & \bar{q}_{2} \\
p_{1} & p_{2}
\end{array}\right|}{\left|\begin{array}{ll}
1 & 1 \\
p_{1} \bar{q}_{1} & p_{2} \bar{q}_{2}
\end{array}\right|}\left(\frac{\left|\begin{array}{ll}
1 & 1 \\
p_{1} q_{1} & p_{2} q_{2}
\end{array}\right|}{\left|\begin{array}{ll}
q_{1} & q_{2} \\
p_{1} & p_{2}
\end{array}\right|} M_{1}^{(0)}+\frac{1}{4 \rho} \frac{\left|\begin{array}{ll}
1 & 1 \\
p_{1}^{2} & p_{2}^{2}
\end{array}\right|}{\left|\begin{array}{ll}
q_{1} & q_{2} \\
p_{1} & p_{2}
\end{array}\right|}\right) \text {, }
\end{aligned}
$$

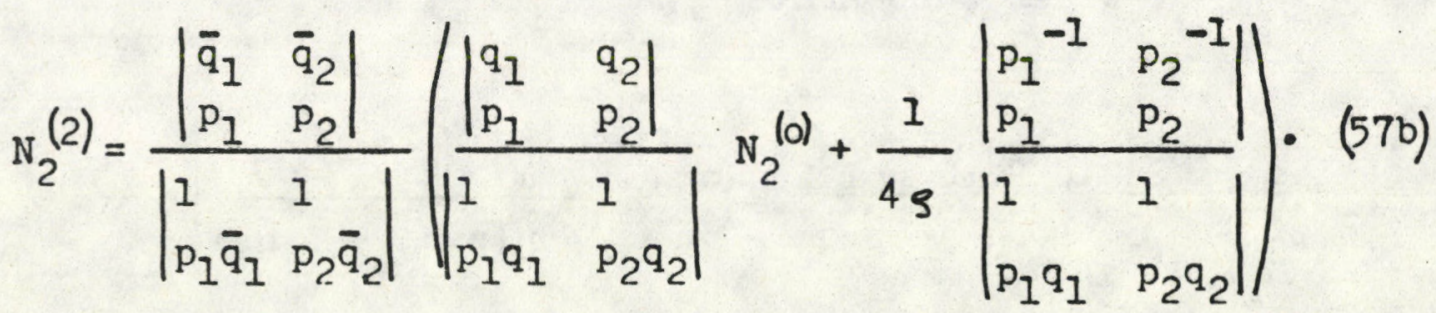

The above form is most useful if we want to generalize it for an arbitrary number of steps. Having calculated the analogous formuIae for 3 B transformations it was quite evident what the corresponding result would be after iterating n-times the $B$ transformation. We realized that these $B$ transformations have the 
intrigueing property that an even or an odd number of iterations should be treated separately. We present here our results in the following compact form, for an even number of steps

$$
\begin{aligned}
& M_{1}^{(2 k)}=\frac{D_{1}^{(2 k)}\left(\bar{q}_{i}\right)}{D_{2}^{(2 k)}\left(\bar{q}_{i}\right)}\left(\frac{D_{2}^{(2 k)}\left(q_{i}\right)}{D_{1}^{(2 k)}\left(q_{i}\right)} M_{1}^{(0)}+\frac{1}{4 \rho} \frac{D_{3}^{(2 k)}\left(q_{i}\right)}{D_{1}^{(2 k)}\left(q_{i}\right)}\right), \\
& N_{2}^{(2 k)}=\frac{D_{1}^{(2 k)}\left(\bar{q}_{i}\right)}{D_{2}^{(2 k)}\left(\bar{q}_{i}\right)}\left(\frac{D_{1}^{(2 k)}\left(q_{i}\right)}{D_{2}^{(2 k)}\left(q_{i}\right)} N_{2}^{(0)}+\frac{1}{4 \rho} \frac{D_{4}^{(2 k)}\left(q_{i}\right)}{D_{2}^{(2 k)}\left(q_{i}\right)}\right),
\end{aligned}
$$

where the $D_{a}$ 's / $a=1, \ldots 4 /$ are $2 k \times 2 k$ determinants and they are completely characterized by their i-th row

$$
\begin{aligned}
& \mathrm{D}_{1}^{(2 k)}\left(\mathrm{q}_{i}\right)=\left|\mathrm{q}_{i}, \mathrm{p}_{i}, \mathrm{p}_{i}^{2} \mathrm{q}_{i}, \mathrm{p}_{i}^{3}, \mathrm{p}_{i}^{4} \mathrm{q}_{i}, \ldots ., \mathrm{p}_{i}{ }^{2 \mathrm{k}-1}\right| \\
& \mathrm{D}_{2}^{(2 \mathrm{k})}\left(\mathrm{q}_{i}\right)=\left|\mathrm{l}, \mathrm{p}_{i} \mathrm{q}_{i}, \mathrm{p}_{i}^{2}, \mathrm{p}_{i}^{3} \mathrm{q}_{i}, \mathrm{p}_{i}^{4}, \ldots ., \mathrm{p}_{i}{ }^{2 \mathrm{k}-\mathrm{l}} \mathrm{q}_{i}\right| \\
& \mathrm{D}_{3}{ }^{(2 \mathrm{k})}\left(\mathrm{q}_{\mathrm{i}}\right)=\left|1, \mathrm{p}_{i} \mathrm{q}_{i}, \mathrm{p}_{\mathrm{i}}^{2}, \mathrm{p}_{\mathrm{i}}^{3} \mathrm{q}_{\mathrm{i}}, \mathrm{p}_{i}^{4}, \ldots ., \mathrm{p}_{\mathrm{i}}^{2 \mathrm{k}-2}, \mathrm{p}_{\mathrm{i}}^{2 \mathrm{k}}\right| \\
& \mathrm{p}_{4}^{(2 k)}\left(\mathrm{q}_{i}\right)=\left|\mathrm{p}_{i}^{-1}, \mathrm{p}_{i}, \mathrm{p}_{i}^{2} \mathrm{q}_{i}, \mathrm{p}_{i}^{3}, \mathrm{p}_{i}^{4} \mathrm{q}_{i}, \ldots, \mathrm{p}_{i}^{2 \mathrm{k}-1}\right|
\end{aligned}
$$

and $i=1, \ldots, 2 \mathrm{n}$;

for an odd number of steps

$$
M_{1}^{(2 k+1)}=-\frac{D_{1}^{(2 k+1)}\left(\bar{q}_{i}\right)}{D_{2}^{(2 k+1)}\left(\bar{q}_{i}\right)}\left(\frac{D_{2}^{(2 k+1)}\left(q_{i}\right)}{D_{1}^{(2 k+1)}\left(q_{i}\right)} M_{2}^{(0)}+\frac{1}{4 \rho} \frac{D_{3}^{(2 k+1)}\left(q_{i}\right)}{D_{1}^{(2 k+1)}\left(q_{i}\right)}\right) \text {, }
$$




$$
N_{2}{ }^{(2 k+1)}=-\frac{D_{1}^{(2 k+1)}\left(\bar{q}_{i}\right)}{D_{2}^{(2 k+1)}\left(\bar{q}_{i}\right)}\left(\frac{D_{1}^{(2 k+1)}\left(q_{i}\right)}{D_{2}^{(2 k+1)}\left(a_{i}\right)} N_{2}^{(0)}+\frac{1}{4 \rho} \frac{D_{4}^{(2 k+1)}\left(q_{i}\right)}{D_{2}^{(2 k+1)}\left(a_{i}\right)}\right),
$$

here $D_{\dot{a}}^{\prime}$ are $(2 k+1) \times(2 k+1)$ determinants written in an analogous way as above

$$
\begin{aligned}
& D_{1}^{(2 k+1)}\left(q_{i}\right)=\left|q_{i}, p_{i}, p_{i}^{2} q_{i}, p_{i}^{3}, p_{i}^{4} q_{i}, \ldots, \ldots, p_{i}^{2 k} q_{i}\right| \\
& D_{2}^{(2 k+1)}\left(q_{i}\right)=\left|1, p_{i} q_{i}, p_{i}^{2}, p_{i}^{3} q_{i}, p_{i}^{4}, \ldots, p_{i}^{2 k}\right| \\
& D_{3}^{(2 k+1)}\left(q_{i}\right)=\left|1, p_{i} q_{i}, p_{i}^{2}, p_{i}^{3} q_{i}, p_{i}^{4}, \ldots \ldots, p_{i}^{2 k-1} q_{i}, p_{i}^{2 k+1} q_{i}\right| \\
& \mathrm{p}_{4}^{(2 \mathrm{k}+1)}\left(\mathrm{q}_{i}\right)=\left|\mathrm{p}_{i}^{-1}, \mathrm{p}_{i}, \mathrm{p}_{i}^{2} \mathrm{q}_{i}, \mathrm{p}_{i}^{3}, p_{i}^{4} \mathrm{q}_{i}, \ldots, \mathrm{p}_{i}{ }^{2 \mathrm{k}} \mathrm{q}_{i}\right|
\end{aligned}
$$

where $i=1, \ldots \ldots, 2 \mathrm{k}+1$.

We give an inductive proof of these results in Appendix B.

Formulae (58) and (59) are of fundamental importance since they enable us to construct the explicit $\mathrm{n}$ monopole solutions by pure algebra. In fact, they represent the nonlinear superposition rule. It is a very attractive feature of our method that we get as an output: just the components of the vector potential, and the physically most relevant quantity, the length of the Higgs field $|\phi|$, can be read off immediately. It should be noted here, that Neugebauer [34] has given a compact formul for the Ernst potential, $\epsilon$, for a 2 -fold Harrison transformation. Needless to say, it would be very awkward to use this result in 
our case.

We guarantee the reality of our solutions by imposing the following conditions for $q_{i}$ and $p_{i}$

$$
\mathrm{q}_{2 \mathrm{r}-1}^{*}=\mathrm{q}_{2 \mathrm{r}}^{-1}, \mathrm{p}_{2 \mathrm{r}-1}^{*}=\mathrm{p}_{2 \mathrm{r}}^{-1}
$$

for an even number of steps, whereas for an odd number of iterations

$$
\begin{aligned}
& \mathrm{q}_{1}^{*}=\mathrm{q}_{1}, \mathrm{p}_{1}^{*}=\mathrm{p}_{1}^{-1}, \\
& \mathrm{q}_{2 \mathrm{r}}^{*}=\mathrm{q}_{2 \mathrm{r}+1}, \mathrm{p}_{2 \mathrm{r}}^{*}=\mathrm{p}_{2 \mathrm{r}+1}^{-1},
\end{aligned}
$$

$r=1, \ldots \ldots, k$. There exist other possibilities as well, however, as it is clear from Ref. [35] the physically interesting case for us is either $(60 a)$ or $(60 b)$. We made the tacit assumption in (60b). that for the seed solution $\psi_{0}=0$

As $|\phi|^{2}$ is the most interesting quantity for us we find it useful to present here.

$$
\begin{aligned}
|\phi|=2 \mid \frac{D_{2}^{(2 k+\epsilon)}\left(q_{i}\right)}{D_{1}^{(2 k+\epsilon)}\left(q_{i}\right)} M_{1+\epsilon}^{(0)}-\frac{D_{1}^{(2 k+\epsilon)}\left(q_{i}\right)}{D_{2}^{(2 k+\epsilon)}\left(q_{i}\right)} N_{2}^{(0)}+ \\
+\frac{1}{4 \rho}\left(\frac{D_{3}^{(2 k+\epsilon)}\left(q_{i}\right)}{D_{1}^{(2 k+\epsilon)}\left(q_{i}\right)}-\frac{D_{4}^{(2 k+\epsilon)}\left(q_{i}\right)}{D_{2}^{(2 k+\epsilon)}\left(q_{i}\right)}\right) \mid
\end{aligned}
$$

Where $\epsilon=0$ or $1, k$ is any integer and we used the reality $(60 a)$ or $(60 b)$ respectively. 
To actually get multimonopole configurations one should start with a suitable seed solution for which one has to solve eq. (40) to obtain q. With these q's and the corresponding p's where the parameters $\beta$ 's and w's are arbitrary / up to the constraints (60) / one usually ends up with singularities in

$|\phi|$, therefore, the energy for this configuration will not be in general finite. As it was found in [35] the parameters $\beta_{i}, w_{i}$ are uniquely determined by imposing the condition of finite energy. In fact this constraint means that there be no singularities in eq. (61) for $|\phi|$.

7. GENERATING MULTIMONOPOLES

We now show how can one make the first step in applying the method developped in this paper which amounts to generate the singly charged monopole. We indicate here why the modified BT, $\mathrm{B} /=\mathrm{IH} /$, was singled out as the candidate for generating the nonlinear superposition of monopoles.

The most naive expectation is that one could start with the well known BPS one monopole / IMP / as seed solution and applying a BT one would end up with a doubly charged monopole, etc. In the Sine-Gordon theory this is indeed the case, that is from a soliton a BT generates a two soliton, etc. In practice it is not quite straightforward to proceed this way since the IMP is somewhat complicated in the R gauge; its Ernst potential, $\epsilon_{\text {MMP }}$ 
is given by $[10,29] \quad \epsilon=F^{-1}(\rho+i P)$, where

$$
\begin{aligned}
& F=\frac{r}{\sinh r}+r \cosh z \operatorname{coth} r-z \sinh z, \\
& P=z \cosh z-r \sinh z \operatorname{coth} z
\end{aligned}
$$

It is not an easy task to solve eq. (40) for $q_{\text {IMP }}$ so one should find an other way. It is well known in other models that the one soliton solution can be reached from the vacuum state by a BT. In our case the "vacuum" is not the trivial solution of the Erngt equation, $f=1, \quad \psi=0$ which corresponds to Minkowski space, but rather a more "natural" vacuum would be the Higgs vacuum, $\phi^{2}=1$. The simplest solution of the Ernst equation with this property is $f_{0}=e^{z}, \psi_{0}=0$, or any Ehlers transformation of $f_{0}$. As it was indicated in Ref. [10] a simple HBT does not give the IMP from this "vacuum set". Instead, it was found that the HI transformation gives rise to the IMP when applied to the complex solution $f^{\prime}=\frac{1}{\sinh z}, \quad \psi^{\prime}=i \operatorname{coth} z$, which is obtained from $\mathrm{e}^{\mathrm{z}}$ by a complex Ehlers transformation. Even for this complex vacuum state the solution of eq. (40) is quite complicated, so it was important to note that by the $B$ transformation one obtains the IMP just from $f_{0}$. The $M_{i}^{(0)} s, N_{i}^{(0)}, s$ for $f_{0}=e^{z}$, $\psi_{0}=0$ are given by $M_{1}^{(0)}=M_{2}^{(0)}=-\frac{i}{4}, N_{1}^{(0)}=N_{2}^{(0)}=\frac{i}{4}$. Eq. (40) is readily integrated and we get for $q$

$$
q=-\tanh (R(w)-\beta)
$$


where $R(w)=\sqrt{(w-z)^{2}+\rho^{2}}$ and $\beta$ is the constant of integration. Using eqs. (47) and (51) we obtain for $\phi^{2}$

$$
\phi^{2}=\left(\operatorname{coth} R(w)-\frac{1}{R(w)}\right)^{2}
$$

where we had to choose $\beta=0$ to avoid singularities and we used

$$
\left|\frac{p+q}{I+p q}\right|=I
$$

One would now think the way is open to apply a second B transformation for the IMP to get a 2MP, etc. However, in contrast to the known cases it is impossible to generate the 2MP from the IMP by a BT / either by B or HBT /. The reason of this surprising fact will become clearer if we see how the 2MP can be generated. The "vacuum" we start with is again $f_{0}=e^{z}$ and the constants we had to choose to ensure the absence of singularities in $|\phi|$ are: $w_{1}=w_{2}^{*}=i \frac{\pi}{2}, \beta_{1}=0, \quad \beta_{2}=-i \frac{\pi}{2} \cdot|\phi|$ can now be calculated after some algebra [9]

$$
\begin{aligned}
& \left.|\phi|^{2}=\right]^{-1}\left\{\left[\left(1+\eta^{2}\right) \cos \alpha \xi\left(\alpha\left[\xi^{2}+\eta^{2}\right] \cos \alpha \xi-2 \xi \sin \alpha \xi\right)+\right.\right. \\
+ & \left.\left(1-\xi^{2}\right) \cosh \alpha \eta\left(\alpha\left[\xi^{2}+\eta^{2}\right] \cosh \alpha \eta-2 \eta \sinh \alpha \eta\right)\right]^{2}+ \\
+ & \left.4\left[\xi\left(1+\eta^{2}\right) \cos \alpha \xi \sinh \alpha \eta-\eta\left(1-\xi^{2}\right) \cosh \alpha \eta \sin \alpha \xi\right]^{2}\right\}
\end{aligned}
$$

where 


$$
\exists=\alpha^{2}\left(\xi^{2}+\eta^{2}\right)^{2}\left[\left(1-\xi^{2}\right) \cosh ^{2} \alpha \eta-\left(1+\eta^{2}\right) \cos ^{2} \alpha \xi\right]^{2}, \alpha=\frac{\pi}{2}
$$

and we introduced oblate spheroidal coordinates

$$
\sqrt{\left(1-\xi^{2}\right)\left(1+\eta^{2}\right)}+i \xi \eta=\frac{\rho+i z}{\alpha} ;-1 \leq \xi \leq 1 ; 0 \leq \eta \leq \infty
$$

$|\phi|$ drastically simplifies on the $z=0$ plane and on the $z$ axis

$$
\left|\phi\left(z=0, \rho_{\alpha}\right)\right|=\left|1+\frac{2 \alpha^{2} \cosh \rho_{\alpha}\left(\rho_{\alpha} \cosh \rho_{\alpha}-\sinh \rho_{\alpha}\right)}{\rho_{\alpha}\left(\rho_{\alpha}^{2}-\alpha^{2} \sinh \rho^{2} \rho_{\alpha}\right)}\right|
$$

where $\rho_{\alpha}=\sqrt{\rho^{2}-\alpha^{2}}$

$$
|\phi(z, \rho=0)|=\left|\tanh z-\frac{2 z}{\alpha^{2}+z^{2}}\right|
$$

This solution describes a doubly charged monopole situated at the origin.

Thus we see that the 2MP is generated by a double B transformation, as we claimed, but the second of these transformations is carried out on a state entirely different from the IMP.

Finally, we show that applying (61) to the simplest Higgs vacuum the asymptotic behaviour of $|\phi|$ satisfies eq. (8) . First we observe that $q$ of eq. (63) tends to -1 exponentially 
for $r \rightarrow \infty$ while $|p|$ approaches $I$ here only polynomially . Therefore keeping in (6I) the polynomial terms only we get

$$
\frac{D_{1}^{(2 k+\epsilon)}}{D_{2}^{(2 k+\epsilon)}}=(-1)^{\epsilon}
$$

while using an identity [36] for the ratio of two $\forall a n d e r m o n d e-$ type determinants

$$
\frac{D_{3}^{(n)}}{D_{1}^{(n)}}=(-1)^{n+1} \sum_{i=1}^{n} p_{i}, \frac{D_{4}^{(n)}}{D_{2}^{(n)}}=(-1)^{n+1} \sum_{i=1}^{n} \frac{1}{p_{i}} .
$$

Now plugging these ratios as well as $M_{i}^{(0)}=-\frac{i}{4}, N_{i}^{(0)}=\frac{i}{4}$ into (61) we obtain

$$
|\phi| \underset{\gamma \rightarrow \infty}{\sim}\left(1-\sum_{i=1}^{n} \frac{1}{R\left(w_{i}\right)}\right) \sim\left(1-\frac{n}{r}\right) .
$$

This shows that every $B$ transformation increases the topological charge by one. However, the resulting configurations are in general singular, corresponding to infinite energy Therefore, the only remaining task is to find those values of $w_{i}, \beta_{i}$ for which $|\phi|$ given by $(61)$ is nonsingular. This was achieved in Ref. [35], where the structure of these new solutions is thoroughly discussed. 


\section{CONCLUSIONS}

We derived the general form of the $n$-fold BT for the axially and mirror symmetric Bogomolny equations. We have pointed out that applying these BT's on simple Higgs vacuum one can generate monopoles of arbitrary charge. We illustrated our method by showing how the one monopole and a new doubly charged monopole emerges from this process.

After our work was completed, we received the papers by Prasad, Sinha, Wang $[37]$ in which they described another framework to generate multimonopoles, generalizing Ward's construction. From the comparison of these two superficially very different methods we can immediately establish that our BT connects the $A_{l}$ and the $A_{\ell+1}$ ansätze while preserving reality and regularity properties of the seed solution / at least for a suitable choice of the parameters /. 


\section{APPENDIX A. COMPOSITION THEOREM}

We give a detailed derivation of the composition theorem for two subsequent BT's.

The Riccati equation (34) for the pseudopotential, q', in the second $\mathrm{BT}$ is

$$
\begin{aligned}
d^{\prime}= & {\left[-N_{1} \frac{q_{1}}{\bar{q}_{1}} q^{\prime}\left(I+p_{2} q^{\prime}\right)+M_{2} \frac{\bar{q}_{1}}{q_{1}}\left(q^{\prime}+p_{2}\right)+\right.} \\
& \left.+\frac{p_{1}^{2}-1}{4 \rho}\left(q_{1} q^{\prime}\left(I+p_{2} q^{\prime}\right)-\left(q^{\prime}+p_{2}\right)\right)\right] d \zeta_{1}+ \\
& +\left[-q_{1} \bar{q}_{1} N_{2}\left(I+\frac{q^{\prime}}{p_{2}}\right) q^{\prime}+N_{1} \frac{1}{q_{1} \bar{q}_{1}}\left(q^{\prime}+\frac{1}{p_{2}}\right)+(A \cdot I)\right. \\
& \left.+\frac{I-p_{1}^{2}}{4 \rho p_{1}}\left(\frac{q_{1} q^{\prime}}{I+p_{1} q_{1}}\left(I+\frac{q^{\prime}}{p_{2}}\right)-\frac{1}{p_{1}+q_{1}}\left(q^{\prime}+\frac{1}{p_{2}}\right)\right)\right] a \zeta_{2}
\end{aligned}
$$

From the assumption $q^{\prime}=q^{\prime}\left(p_{1}, p_{2}, q_{1}, q_{2}\right)$ we get for $d q^{\prime}$

$$
\mathrm{dq}^{\prime}=\frac{\partial q^{\prime}}{\partial q_{1}} d q_{1}+\frac{\partial q^{\prime}}{\partial q_{2}} d q_{2}+\frac{\partial q^{\prime}}{\partial p_{1}} d p_{1}+\frac{\partial q^{\prime}}{\partial p_{2}} d p_{2} \cdot(A .2)
$$

Now substituting $\mathrm{dq}_{1}$ and $\mathrm{dq}_{2}$ from eq. (34) and since

$$
d p_{i}=\frac{p_{i}^{2}-I}{4 \rho}\left[p_{i} d \zeta_{1}+\frac{I}{p_{i}} d \zeta_{2}\right] \text {, }
$$


$-37-$

we get comparing (A.I) and (A.2) the foll owing equations for q'

$$
\begin{aligned}
& \frac{\partial q^{\prime}}{\partial q_{1}} q_{1}\left(I+p_{1} q_{1}\right)+\frac{\partial q^{\prime}}{\partial q_{2}} q_{2}\left(I+p_{2} q_{2}\right)=q^{\prime} \frac{q_{1}}{\bar{q}_{1}}\left(1+p_{2} q^{\prime}\right),(A \cdot 4 a) \\
& \frac{\partial q^{\prime}}{\partial q_{1}}\left(q_{1}+p_{1}\right)+\frac{\partial q^{\prime}}{\partial q_{2}}\left(q_{2}+p_{2}\right)=\frac{\bar{q}_{1}}{q_{1}}\left(q^{\prime}+p_{2}\right) \quad,(A .4 b) \\
& \frac{\partial q^{\prime}}{\partial q_{1}} q_{1}\left(1+\frac{q^{\prime}}{p_{1}}\right)+\frac{\partial q^{\prime}}{\partial q_{2}} q_{2}\left(1+\frac{q_{2}}{p_{2}}\right)=q_{1} \bar{q}_{1}\left(1+\frac{q^{\prime}}{p_{2}}\right) q^{\prime}, \quad(A \cdot 4 c) \\
& \frac{\partial q^{\prime}}{\partial q_{1}}\left(q_{1}+\frac{1}{p_{1}}\right)+\frac{\partial q^{\prime}}{\partial q_{2}}\left(q_{2}+\frac{1}{p_{2}}\right)=\frac{1}{q_{1} \bar{q}_{1}}\left(q^{\prime}+\frac{1}{p_{2}}\right) \quad, \text { (A.4d) } \\
& \frac{\partial q^{\prime}}{\partial p_{1}} p_{1}\left(p_{1}^{2}-1\right)+\frac{\partial q^{\prime}}{\partial p_{2}} p_{2}\left(p_{2}^{2}-1\right)=\left(p_{1}^{2}-1\right)\left[q_{1} q^{\prime}\left(1+p_{2^{\prime}} q^{\prime}\right)-\left(q^{\prime}+p_{2}^{\prime}\right)\right], \\
& \frac{\partial q^{\prime}}{\partial p_{1}} \frac{p_{1}^{2}-1}{p_{1}}+\frac{\partial q^{\prime}}{\partial p_{2}} \frac{p_{2}^{2}-1}{p_{2}}= \\
& =\frac{I-p_{1}^{2}}{p_{1}}\left[\frac{q_{1} q^{\prime}}{I+p_{1} q_{1}}\left(1+\frac{q^{\prime}}{p_{2}}\right)-\frac{1}{p_{1}+q_{1}}\left(q^{\prime}+\frac{1}{p_{2}}\right)\right]
\end{aligned}
$$

We see that we got an overdetermined system of differential equaltrons for $q^{\prime}$, and it is possible to reduce eq. (A.4Q-d) just 
to simple algebraic equations

$$
\begin{aligned}
& \frac{\bar{q}_{1} q_{2}-\bar{q}_{2} q_{1}}{q_{1} \bar{q}_{1}-q_{2} \bar{q}_{2}}=\frac{q_{1} q^{\prime}-\bar{q}_{1} \bar{q}^{\prime}}{1-q_{1} \bar{q}_{1} q^{\prime} \bar{q}^{\prime}}, \\
& \frac{p_{1}}{p_{2}} \frac{\bar{q}_{1} q_{2}-\bar{q}_{2} q_{1}}{q_{1} \bar{q}_{1}-q_{2} \bar{q}_{2}}=\frac{\frac{q}{1}_{1}^{2} \bar{q}_{2} q^{\prime}-\bar{q}_{1}^{2} q_{2} \bar{q}^{\prime}}{q_{2} \bar{q}_{2}-q_{1}^{2} \bar{q}_{1}^{2} q^{\prime} \bar{q}^{\prime}} .
\end{aligned}
$$

These two quadratic equations for $q^{\prime} /$ or $\bar{q}^{\prime} /$ are easily solved and indeed they have one common root, or alternatively one can reduce $(A .5 a-b)$ to a linear equation, the solution of which is seen to satisfy both $(\mathrm{A} \cdot 5 \mathrm{a}-\mathrm{b})$.

$$
q^{\prime}=\frac{1}{q_{1}} \frac{\bar{q}_{1} p_{2}-\bar{q}_{2} p_{1}}{\bar{q}_{1} p_{1}-\bar{q}_{2} p_{2}}
$$

$I^{\prime}$ ' $=\vec{q}$ ' is the "dual" of $q^{\prime}$ in the sense that one should simply replace $\bar{q}_{1}, \bar{q}_{2}$ and $q_{1}$ in $(\AA .6)$ by $q_{1}, q_{2}$ and $\bar{q}_{1}$. One can verify that (A.6) really solves all of the eqs. in (A.4). This $q^{\prime}$ satisfies eq. (34) with $p==p_{2}$ and $M_{i}, N_{i}$ replaced by $H\left(q_{1}, p_{1}\right) M_{i}, H\left(q_{1}, p_{1}\right) N_{i}$. 
APPENDIX B. PROOF OF N-STEP FORMULAE

We proceed to prove our results for iterating an arbitrary number of B transformations given by formulae (58) and (59), using the method of induction.

The validity of (58) and (59) in the case when $k=1$ and 2 respectively / corresponding to $2 \mathrm{MP}$ and the IMP / can be established without any difficulty.

Let us now suppose that our formulae have been verified up to $2 \mathrm{k}$ steps. We show that doing one more step we indeed recover (59) corresponding to $2 \mathrm{k}+\mathrm{I}$ steps. In practice it is more tractable to do it in the other way round, that is to make first a single $B$ transformation / by $q_{1}$ and $p_{1} /$, then apply the assumption for $2 \mathrm{k}$ steps which gives

$$
\begin{aligned}
& M_{1}^{(2 k+1)}=\frac{D_{1}^{(2 k)}\left(q_{j}^{j}\right)}{D_{2}^{(2 k)}\left(q_{j}^{\prime}\right)}\left(\frac{D_{2}^{(2 k)}\left(\bar{q}_{j}^{\prime}\right)}{D_{1}^{(2 k)}\left(\bar{q}_{j}^{\prime}\right)} M_{1}^{(1)}+\frac{1}{4 \rho} \frac{D_{3}^{(2 k)}\left(\bar{q}_{j}^{\prime}\right)}{D_{1}^{(2 k)}\left(\bar{q}_{j}^{\prime}\right)}\right), \\
& N_{2}^{(2 k+1)}=\frac{D_{1}^{(2 k)}\left(q_{j}^{\prime}\right)}{D_{2}^{(2 k)}\left(q_{j}^{j}\right)}\left(\frac{D_{1}^{(2 k)}\left(\bar{q}_{j}^{\prime}\right)}{D_{2}^{(2 k)}\left(\bar{q}_{j}^{\prime}\right)} N_{2}^{(1)}+\frac{1}{4 \rho} \frac{D_{4}^{(2 k)}\left(\bar{q}_{j}^{\prime}\right)}{D_{2}^{(2 k)}\left(\bar{q}_{j}^{j}\right)}\right),
\end{aligned}
$$

where $2 \leqslant j \leqslant 2 k+1$, and

$$
q_{j}^{\prime} q_{1}=\frac{p_{j} \bar{q}_{I}-p_{1} \bar{q}_{j}}{p_{1} \bar{q}_{1}-p_{j} \bar{q}_{j}}, \quad \bar{q}_{j}^{\prime} \bar{q}_{1}=\frac{p_{j} q_{1}-p_{1} q_{j}}{p_{1} q_{I}-p_{j} q_{j}} \text {. }
$$


We recast the ratios of the determinants, $D_{\alpha} / \alpha=1, \ldots, 4 /$ using (B.2) in the following form

$$
\begin{aligned}
& \frac{D_{1}^{(2 k)}\left(q_{j}^{\prime}\right)}{D_{2}^{(2 k)}\left(q_{j}^{\prime}\right)}=\frac{D_{1}^{(2 k)}\left(q_{j}^{\prime} q_{1}\right)}{D_{2}^{(2 k)}\left(q_{j}^{\prime} q_{1}\right)}=\frac{T_{1}\left(\bar{q}_{j}\right)}{T_{2}\left(\bar{q}_{j}\right)}, \frac{D_{1}^{(2 k)}\left(\bar{q}_{j}^{\prime}\right)}{D_{2}^{(2 k)}\left(\bar{q}_{j}^{\prime}\right)}=\frac{T_{1}\left(q_{j}\right)}{T_{2}\left(q_{j}\right)}, \\
& \frac{D_{3}^{(2 k)}\left(\bar{q}_{j}^{\prime}\right)}{D_{1}^{(2 k)}\left(\bar{q}_{j}^{\prime}\right)}=\bar{q}_{j} \frac{T_{3}\left(q_{j}\right)}{T_{1}\left(q_{j}\right)}, \quad \frac{D_{4}^{(2 k)}\left(\bar{q}_{j}^{\prime}\right)}{D_{2}^{(2 k)}\left(\bar{q}_{j}^{\prime}\right)}=\bar{q}_{j} \frac{T_{4}\left(q_{j}\right)}{T_{2}\left(q_{j}\right)},
\end{aligned}
$$

where the $T_{\alpha}$ 's are $2 \mathrm{k} \times 2 \mathrm{k}$ determinants defined as

$$
\begin{aligned}
& T_{1}\left(q_{j}\right)=\mid p_{j} q_{I}-p_{1} q_{j}, p_{j}\left(p_{I} q_{I}-p_{j} q_{j}\right), p_{j}^{2}\left(p_{j} q_{I}-p_{1} q_{j}\right), \ldots \\
& \ldots, p_{j}^{2 k-1}\left(p_{1} q_{1}-p_{j} q_{j}\right) \mid \text {, } \\
& T_{2}\left(q_{j}\right)=\mid p_{1} q_{1}-p_{j} q_{j}, p_{j}\left(p_{j} q_{1}-p_{1} q_{j}\right), \quad p_{j}^{2}\left(p_{1} q_{1}-p_{j} q_{j}\right), \ldots \\
& \ldots, p_{j}^{2 k-1}\left(p_{j} q_{1}-p_{1} q_{j}\right) \mid \text {, } \\
& T_{3}\left(q_{j}\right)=\mid p_{I} q_{I}-p_{j} q_{j}, p_{j}\left(p_{j} q_{I}-p_{I} q_{j}\right), \quad p_{j}^{2}\left(p_{1} q_{1}-p_{j} q_{j}\right), \ldots \\
& \ldots, p_{j}^{2 k-2}\left(p_{1} q_{1}-p_{j} q_{j}\right), p_{j}^{2 k}\left(p_{1} q_{1}-p_{j} q_{j}\right) \mid \quad \text {, (B.4c) } \\
& T_{4}\left(q_{j}\right)=\mid p_{j}^{-1}\left(p_{1} q_{1}-p_{j} q_{j}\right), p_{j}\left(p_{1} q_{1}-p_{j} q_{j}\right), p_{j}^{2}\left(p_{j} q_{1}-p_{1} q_{j}\right), \ldots \\
& \cdots, p_{j}^{2 k-1}\left(p_{1} q_{1}-p_{j} q_{j}\right) \mid \text {, }
\end{aligned}
$$

(B.la,b) are rewritten without difficulty using the $T_{\alpha}$ 's

$$
M_{1}^{(2 k+1)}=-\bar{q}_{1} \frac{T_{1}\left(\bar{q}_{j}\right)}{T_{2}\left(\bar{q}_{j}\right)}\left[\frac{T_{2}\left(q_{j}\right)}{T_{1}\left(q_{j}\right)} \frac{1}{q_{j}} M_{2}^{(0)}+\frac{1}{4 \rho} \frac{p_{1} T_{2}\left(q_{j}\right)-T_{3}\left(q_{j}\right)}{T_{1}\left(q_{j}\right)}\right] \text {, }
$$




$$
N_{2}^{(2 k+1)}=-\bar{q}_{1} \frac{T_{1}\left(\bar{q}_{j}\right)}{T_{2}\left(\bar{q}_{j}\right)}\left[\frac{T_{1}\left(q_{j}\right)}{T_{2}\left(q_{j}\right)} q_{1} N_{2}^{(0)}+\frac{1}{4 \rho} \frac{p_{1}^{-T_{1}}\left(q_{j}\right)-T_{4}\left(q_{j}\right)}{T_{2}\left(q_{j}\right)}\right] \text {, }
$$

We shall now write the determinants, $\mathrm{T}_{\alpha}$, in a more useful form, namely as a sum of $2^{2 \mathrm{k}}$ determinants with elements $\mathrm{p}_{1}, \mathrm{p}_{j}, \mathrm{p}_{1} \mathrm{p}_{j}$, etc. To make our proof as transparent as possible, we introduce the following compact notation: let us split the set of indices

$$
\{2,3, \ldots, 2 \mathrm{k}+1\} \text { into two disjoint sets, }\left\{A_{r}\right\} \text { and }\left\{B_{2 k-r}\right\} \text {, }
$$
where $\left\{A_{r}\right\}$ contains $r$ different indices and $\left\{B_{2 k-r}\right\}$ contains the rest. We also introduce $a \in\left\{A_{r}\right\}, b \in\left\{B_{2 k-r}\right\}$ to denote the corresponding elements of these sets and

$$
|a|=\sum_{a \in\left\{A_{r}\right\}} a \quad ; \quad|b|=\sum_{b \in\left\{B_{2 k-r}\right\}} b \text {. }
$$

With this notation we write the $\mathrm{T}_{\alpha}$ 's as

$$
T_{\alpha}\left(q_{j}\right)=\sum_{r=0}^{2 k} \sum_{\left\{A_{r}\right\}} q_{i}^{\prime}\left[\prod_{b \in\left\{B_{2 k-r}\right\}}\left(-q_{b}\right)\right] S_{\alpha}\left(p_{j},\left\{A_{r}\right\}\right),
$$

where we sum for all possible choices of $r$ different indices; i.e. for all different $\left\{A_{r}\right\}$ sets. The determinants, $S_{\alpha}$, introduced in (B.6) can be characterized by two of their typical rows, one of which contains $p_{1}, p_{a}$ where $a \in\left\{A_{r}\right\}$ and the other contains $p_{1}, p_{b}$, where $b \in\left\{B_{2 k-r}\right\}$. This suggests the following shorthand notation for the $S_{\alpha}$ 's / we do not interchange the original 
order of any row /:

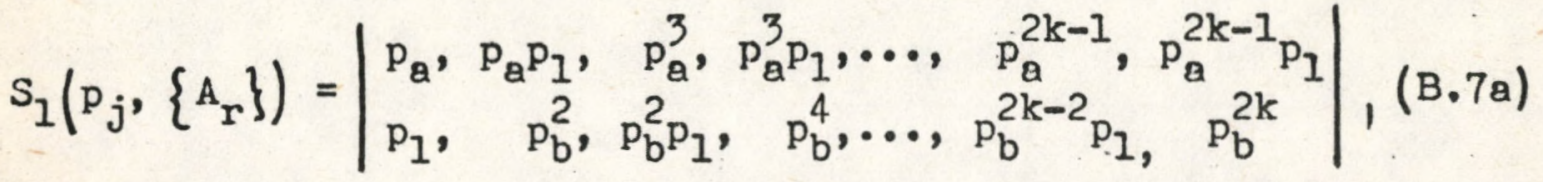

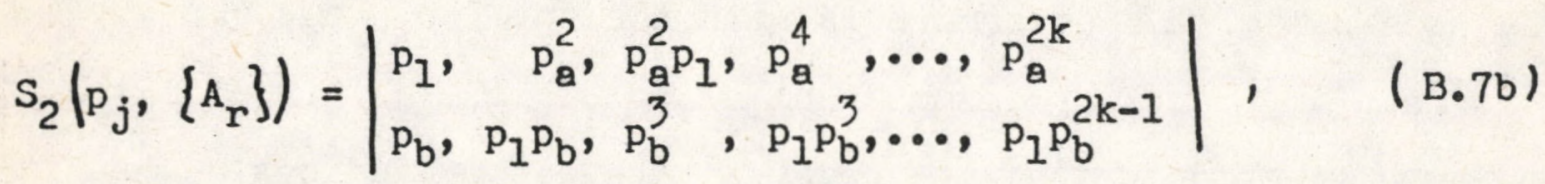

$$
\begin{aligned}
& S_{5}\left(p_{j},\left\{A_{r}\right\}\right)=S_{3}-p_{1} S_{2}=
\end{aligned}
$$

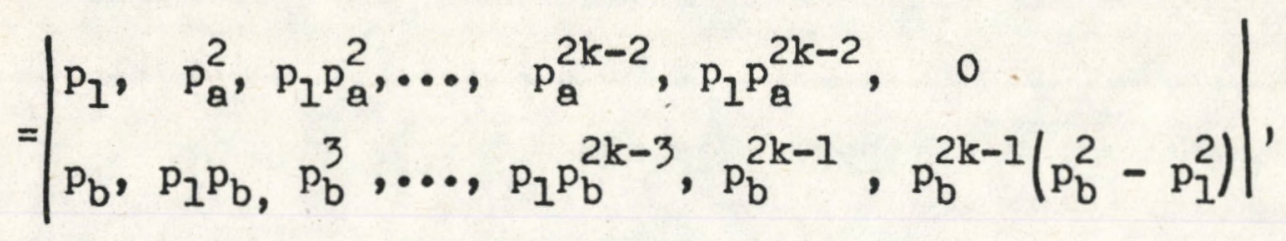

$s_{6}\left(p_{j},\left\{A_{r}\right\}\right)=s_{4}-p_{1}^{-1} s_{1}=$

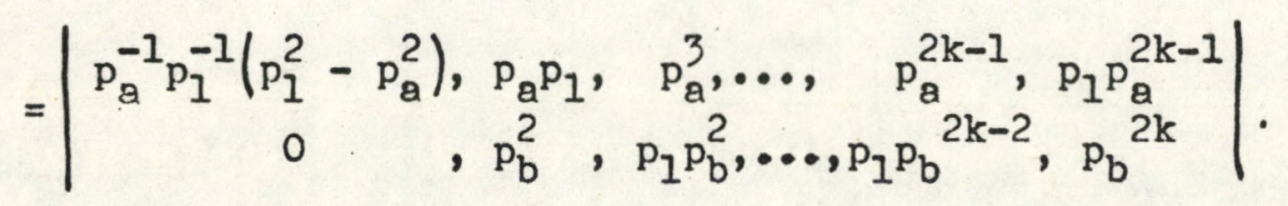

The relevant combinations in (B.5) are in close connection with (B.7) . Now it is very important to realize that most of determinants, $S_{\alpha}\left(p_{j},\left\{A_{r}\right\}\right) \quad$ vanish. We show this explicitly only for $S_{1}$, the argument goes similarly for the others. By algebraic manipulations / linearly combining the columns / we write $S_{1}$ in two different forms 


$$
\begin{aligned}
& S_{1}\left(p_{j},\left\{A_{r}\right\}\right)=
\end{aligned}
$$

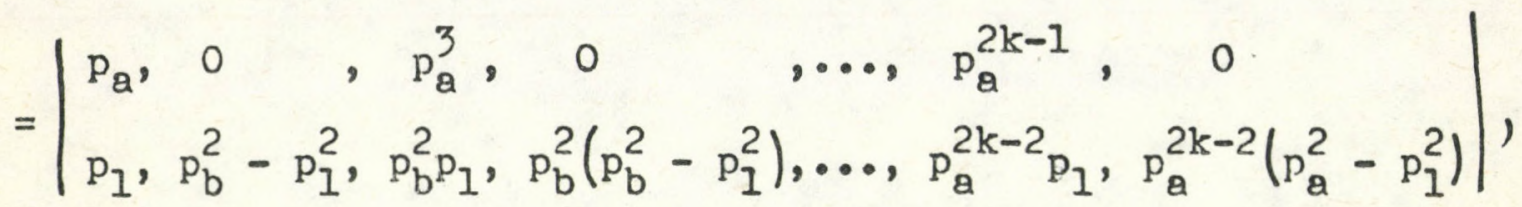

$$
\begin{aligned}
& \text { (B.8a) } \\
& S_{I}\left(p_{j},\left\{A_{r}\right\}\right)=
\end{aligned}
$$

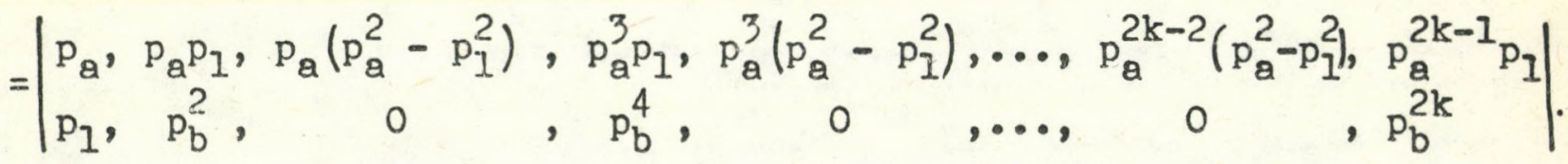

$$
\begin{aligned}
& \text { (B.8b) }
\end{aligned}
$$

It is not difficult to verify that $S_{l}\left(p_{j},\left\{A_{r}\right\}\right)$ is zero for $\mathrm{r}>\mathrm{k} /$ from $(\mathrm{B} 8 \mathrm{a}) /$ and for $\mathrm{r}<\mathrm{k}-\mathrm{l} /$ from(B.8b) /. Similar considerations show that $S_{2}\left(p_{j},\left\{A_{r}\right\}\right)=0$ for $r>k+1$ and $r<k ; s_{5}\left(p_{j},\left\{A_{r}\right\}\right)=0$ for $r>k$ and $r<k-1, s_{6}\left(p_{j},\left\{A_{r}\right\}\right)=$ $=0$ for $r>k+1$ and $r<k$. The only nonvanishing determinants are

$$
\begin{aligned}
& s_{1}\left(p_{j},\left\{A_{k-1}\right\}\right)=(-1)^{|a|}\left[\prod_{a \in\left\{A_{k-1}\right\}}\left(p_{a}^{2}-p_{1}^{2}\right)\right] \cdot p_{1} . \\
& \cdot\left|p_{a}, p_{a}^{3}, \ldots, p_{a}^{2 k-3}\right| \cdot\left|1, p_{b}^{2}, p_{b}^{4}, \ldots, p_{b}^{2 k}\right|,
\end{aligned}
$$


$-44-$

$$
\begin{aligned}
& s_{1}\left(p_{j},\left\{A_{k}\right\}\right)=(-1)^{|a|}\left[\prod_{b \in\left\{B_{k}\right\}}\left(p_{b}^{2}-p_{1}^{2}\right)\right] \text {. } \\
& \cdot\left|p_{a}, p_{a}^{3}, p_{a}^{5}, \ldots, p_{a}^{2 k-1}\right| \cdot\left|1, p_{b}^{2}, p_{b}^{4}, \ldots, p_{b}^{2 k-2}\right| \text {, } \\
& S_{2}\left(p_{j},\left\{A_{k}\right\}\right)=(-1)^{\mid a l+k}\left[\prod_{a \in\left\{A_{k}\right\}}\left(p_{a}^{2}-p_{I}^{2}\right)\right] \text {. } \\
& \cdot\left|1, p_{a}^{2}, p_{a}^{4}, \ldots, p_{a}^{2 k-2}\right| \cdot\left|p_{b}, p_{b}^{3}, \ldots, p_{b}^{2 k-1}\right| \text {, } \\
& S_{2}\left(p_{j},\left\{A_{k+1}\right\}\right)=(-1)^{|a|+k}\left[\prod_{b \in\left\{B_{k-1}\right\}}\left(p_{b}^{2}-p_{l}^{2}\right)\right] \text {. } \\
& \text {, }\left|1, p_{a}^{2}, p_{a}^{4}, \ldots, p_{a}^{2 k}\right| \cdot\left|p_{b}, p_{b}^{3}, p_{b}^{5}, \ldots, p_{b}^{2 k-3}\right| \text {, } \\
& S_{5}\left(p_{j},\left\{A_{k-1}\right\}\right)=(-1)^{|a|+k+1}\left[\prod_{a \in\left\{A_{k-1}\right\}}\left(p_{a}^{2}-p_{1}^{2}\right)\right] \text {. } \\
& \cdot\left|1, p_{a}^{2}, p_{a}^{4}, \ldots, p_{a}^{2 k-4}\right| \cdot\left|p_{b}, p_{b}^{3}, p_{b}^{5}, \ldots, p_{b}^{2 k-1}, p_{b}^{2 k+1}\right| \text {, } \\
& S_{5}\left(p_{j},\left\{A_{k}\right\}\right)=(-I)^{|a|+k+I} p_{I}\left[\prod_{b \in\left\{B_{k}\right\}^{2}}\left(p_{b}^{2}-p_{l}^{2}\right)\right] . \\
& \left|1, p_{a}^{2}, p_{a}^{4}, \ldots, p_{a}^{2 k-2}\right| \cdot\left|p_{b}, p_{b}^{3}, p_{b}^{5}, \ldots, p_{b}^{2 k-1}\right| \text {, } \\
& S_{6}\left(p_{j},\left\{A_{k}\right\}\right)=(-I)^{|a|+I} p_{1}^{-1}\left[\prod_{a \in\left\{A_{k}\right\}}\left(p_{a}^{2}-p_{l}^{2}\right)\right] \text {. } \\
& \left|p_{a}^{-1}, p_{a}, p_{a}^{3}, \ldots, p_{a}^{2 k-3}\right| \cdot\left|p_{b}^{2}, p_{b}^{4}, \ldots, p_{b}^{2 k}\right| \text {, } \\
& s_{6}\left(p_{j},\left\{A_{k+1}\right\}\right)=(-1)^{|a|+1} p_{1}^{2}\left[\prod_{b \in\left\{B_{k-1}\right\}}\left(p_{b}^{2}-p_{1}^{2}\right)\right] \text {. } \\
& \cdot\left|p_{a}^{-1}, p_{a}, p_{a}^{3}, \ldots, p_{a}^{2 k-1}\right| \cdot\left|p_{b}^{2}, p_{b}^{4}, \ldots, p_{b}^{2 k-2}\right| . \text { (B.9) }
\end{aligned}
$$


The next step is to compute the relevant combinations of the $\mathrm{T}_{\alpha}$ 's $/ \mathrm{T}_{1}, \mathrm{~T}_{2}, \mathrm{~T}_{5}, \mathrm{~T}_{6} /$ using $(\mathrm{B} .6)$ and (B.9). The important point in the calculation is to apply the following identity for Vandermonde determinants

$$
\begin{aligned}
\left|1, a_{i}, a_{i}^{2}, \ldots, a_{i}^{k-1}\right|\left[\prod_{i=2}^{k+1}\left(a_{i}-a_{1}\right)\right]= \\
\quad=\left|\begin{array}{ll}
1, a_{1}, a_{1}^{2}, \ldots, a_{1}^{k} \\
1, a_{i}, a_{i}^{2}, \ldots, a_{i}^{k}
\end{array}\right|, \quad 2 \leqslant i \leqslant \cdot k+1 .
\end{aligned}
$$

Finally we get for the T's

$$
\begin{aligned}
& \left(-q_{I}\right)^{-k+l} T_{I}\left(q_{j}\right)= \\
& =\sum_{\left\{A_{k-1}\right\}}(-1)^{|a|}\left|\begin{array}{cccc}
p_{1}, & p_{1}^{3}, & p_{1}^{5}, \ldots, & p_{1}^{2 k-1} \\
p_{a}, & p_{a}^{3}, & p_{a}^{5}, \ldots, & p_{a}^{2 k-1}
\end{array}\right| \cdot\left|q_{b}, p_{b}^{2} q_{b}, p_{b}^{4} q_{b}, \ldots, p_{b}^{2 k} q_{b}\right|+ \\
& +\sum_{\left\{A_{k}\right\}}(-1)^{\mid a l}+1\left|p_{a}, p_{a}^{3}, p_{a}^{5}, \ldots, p_{a}^{2 k-1}\right|\left|\begin{array}{ccc}
q_{1}, & p_{1}^{2} q_{1}, p_{1}^{4} q_{1}, \ldots, p_{1}^{2 k} q_{1} \\
q_{b}, & p_{b}^{2} q_{b}, p_{b}^{4} q_{b}, \ldots, p_{b}^{2 k} q_{b}
\end{array}\right|= \\
& =-D_{1}^{(2 k+1)}\left(q_{i}\right) \\
& \mathrm{q}_{1}^{-\mathrm{k}} \mathrm{T}_{2}\left(\mathrm{q}_{\mathrm{j}}\right)= \\
& =\sum_{\left\{A_{k}\right\}}(-1)^{|a|}\left|\begin{array}{lll}
1, & p_{1}^{2}, p_{1}^{4}, \ldots, p_{1}^{2 k} \\
1, & p_{a}^{2}, p_{a}^{4}, \ldots, p_{a}^{2 k}
\end{array}\right| \cdot\left|p_{b} q_{b}, p_{b}^{3} q_{b}, p_{b}^{5} q_{b}, \ldots, p_{b}^{2 k-1} q_{b}\right|+
\end{aligned}
$$


$-46-$

$$
\begin{aligned}
& +\sum_{\left\{A_{k+1}\right\}}(-1)^{|a|+1}\left|1, p_{a}^{2}, p_{a}^{4}, \ldots, p_{a}^{2 k}\right| \cdot\left|\begin{array}{l}
p_{1} q_{1}, p_{1}^{3} q_{1}, p_{1}^{5} q_{1}, \ldots, p_{1}^{2 k-1} q_{1} \\
p_{b} q_{b}, p_{b}^{3} q_{b}, p_{b}^{5} a_{b}, \ldots, p_{b}^{2 k-1} a_{b}
\end{array}\right|= \\
& =(-1)^{k} D_{2}^{(2 k+1)}\left(a_{i}\right) \\
& q_{1}^{-k+1} T_{5}\left(q_{j}\right)=q_{1}^{-k+1}\left[T_{3}\left(q_{j}\right)-p_{1} T_{2}\left(q_{j}\right)\right]=
\end{aligned}
$$

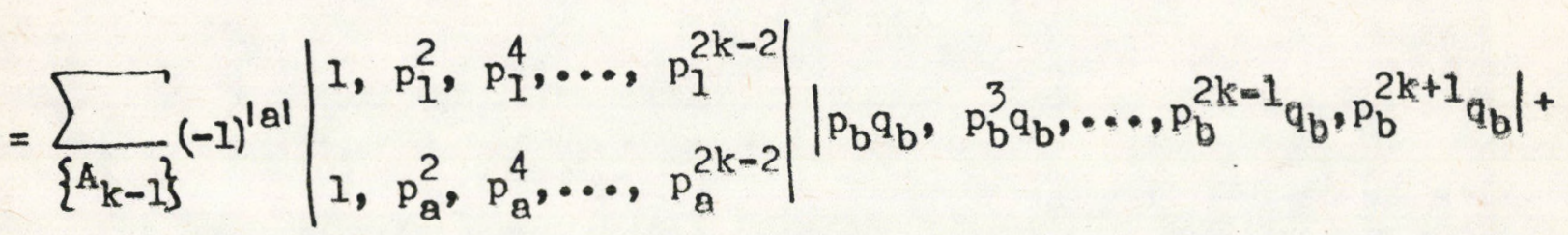

$$
\begin{aligned}
& +\sum_{\left\{A_{k}\right\}}(-1)^{|a|+1}\left|1, p_{a}^{2}, p_{a}^{4}, \ldots, p_{a}^{2 k-2}\right|\left|\begin{array}{c}
p_{1} q_{1}, p_{1}^{3} q_{1}, \ldots, p_{1}^{2 k-1} q_{1}, p_{1}^{2 k+1} q_{1} \\
p_{b} q_{b}, p_{b}^{3} q_{b}, \ldots, p_{b}^{2 k-1} q_{b}, p_{b}^{2 k+1} a_{b}
\end{array}\right|= \\
& =(-1)^{k-1} D_{3}^{(2 k+1)}\left(\mathrm{q}_{i}\right) \\
& \left(-q_{I}\right)^{-k} T_{6}\left(q_{j}\right)=\left(-q_{I}\right)^{-k}\left[T_{4}\left(q_{j}\right)-p_{I}^{-1} T_{I}\left(q_{j}\right)\right]= \\
& =\sum_{\left\{A_{k}\right\}}(-1)^{|a|+1}\left|\begin{array}{lll}
p_{1}^{-1}, & p_{1}, p_{1}^{3}, \ldots, & p_{1}^{2 k-1} \\
p_{a}^{-1}, & p_{a}, p_{a}^{3}, \ldots, & p_{a}^{2 k-1}
\end{array}\right|\left|p_{b}^{2} q_{b}, p_{b}^{4} q_{b}, \ldots, p_{b}^{2 k} q_{b}\right|+ \\
& +\sum_{\left\{A_{k+1}\right\}}(-1)^{|a|}\left|p_{a}^{-1}, p_{a}, p_{a}^{3}, \ldots, p_{a}^{2 k-1}\right|\left|\begin{array}{cc}
p_{1}^{2} q_{1}, & p_{1}^{4} q_{1}, \ldots, p_{1}^{2 k} q_{1} \\
p_{b}^{2} q_{b}, & p_{b}^{4} q_{b}, \ldots, p_{b}^{2 k} q_{b}
\end{array}\right|=
\end{aligned}
$$




$$
=-D_{4}^{(2 k+1)}\left(q_{i}\right)
$$

where $1 \leqslant i \leqslant 2 k+1$. Expanding the determinants, $D{ }_{\alpha}^{(2 k+1)}$, as given in (59c), in terms of their maximal subdeterminants containing only $\mathrm{p}_{i}$ 's yields immediately eqs. ( $\left.\mathrm{B}_{0} 10\right)$. Substituting this result into $(\mathrm{B} .5)$ gives the $2 k+1$ - step formulae what we wanted to prove. Similarly one can obtain the $2 \mathrm{k}+2$ - step formulae starting from the $2 k+1$ - step formulae. 
REFERENCES

[1] G. 'thooft, Nucl. Phys. B79/1974/276;

A.M. Polyakov, JETP Lett. 20 /1974/ 194.

[2] J.C. Pati, A. Salam and J. Strathdee, ICTP-Trieste preprint, IC $80 / 180 / 1980 /$.

[3] M.K. Prasad and C.M. Sommerfield, Phys. Rev。 Lett. 35 /1975/ 760 .

[4] E.B. Bogomolny, Sov. J. Nucl. Phys. $24 / 1976 / 861$.

[5] N.S. Manton, Nucl. Phys. B126/1977/ 525.

[6] W. Nahm, Phys. Lett. 85B /1979/ 373.

[7] C.H. Taubes, Harvard preprint $/ 1980 /$ to be published.

[8] R.S. Ward, Trinity College preprint, Dublin /1980/ to be published.

[9] P. Forgács, Z. Horváth and L. Palla, Phys. Lett. 29B /1981/ 232.

[10] P. Forgács, Z. Horváth and L. Palla, Phys. Rev. Lett. 45 /1980/ 505.

[11] F.J. Ernst, Phys. Rev. $167 / 1968 / 1175$.

[12] B.K. Harrison, Phys. Rev. Lett. 41/1978/ 1197.

[13] G. Neugebauer, J. Phys. A $12 / 1979 /$ L67.

[14] V.A. Belinski and V.E. Zakharov, Sov. Phys. JETP $50 / 1979 / 1$.

[15] E.J. Weinberg and A.H. Guth, Phys。 Rev. D14/1976/ 1660.

[16] P. Forgács and N.S. Manton, Comm. Math. Phys. $72 / 1980 /$

15.

[17] N.S. Manton, Nucl. Phys. B135/1978/ 319. 
[18] W. Kinnersley, J. Math. Phys. /N.1./ 18/1977/ 1529.

[19] C.M. Cosgrove, J.Math. Phys. /N.Y.i 21 /1980/ 2417.

[20] J. Ehlers, in Les Théories Relativistes de la Gravitation, CNRS, Paris, 1959.

[2I] G. Neugebauer and D. Kramer, Ann. Phys / Leipzig / 24 /1969/62.

[22] R. Geroch, J० Math. Phys. /N.Y./ 12 /1971/ 918; J. Math. Phys./N.Y./ $13 / 1972 / 394$.

[23] C. Hoenselaers, W. Kinnersley and B.C. Xanthopoulos,

J. Math. Phys. /N.Y./ $20 / 1979 / 2530$.

[24] D. Maison, J. Math. Phys. /N.Y. / 20 /1979/ 871.

[25] C.N. Yang, Phys. Rev。 Lett. $38 / 1977 / 1377$.

[26] E. Corrigan, D.B. Fairlie, P。Goddard and R.G. Yates, Commun. Math. Phys. 58 /1978/ 223.

[27] L. Witten, Phys。 Rev。 D19/1979/718。

[28] P. Forgács, Z. Horváth and L。 Palla, ITP-Budapest Report

No. 394 /1980/; CRIP preprint, KFKI-1981-06/1981/ to

be published in Phys. Rev. D。

[29] M.A. Lohe, IJucl. Phys. Bl42 /1978/ 236.

[30] E. Corrigan and D.B. Fairlie, Phys. Lett. 67B /1977/ 69;

G. 'tHooft, unpublished;

F. Wilczek, in Quark Confinement and Field Theory / D. Stump and D. Weingarten eds. /, Wiley, N.Y. 1977.

[31] M.F. Atiyah and R.S. Ward, Commun. Math. Phys. 25 /1977/ 117.

[32] R.S. Ward, Phys, Lett. 61A /1977/ 81. 
[33] H.D. Wahlquist and F.B. Estabrook, Phys. Rev. Lett. 21 /1973/ 1386.

[34] G. Neugebauer, J. Phys. A $13 / 1980 /$ L19.

[35] P. Forgács, Z. Horváth and L. Palla, CRIP preprint, Budapest /1981/.

[36] G. Polya and G. Szegס, Problems and Theorems in Analysis, Vol. II.Sect. 8. Springer-Verlag, Berlin-Heidelberg, 1972.

[37] M.K. Prasad, A. Sinha and L.L. Chau Wang, Stony Brook preprint, ITP-SB-80-73/1980/;

M.K. Prasad, MTT preprint /1980/。 



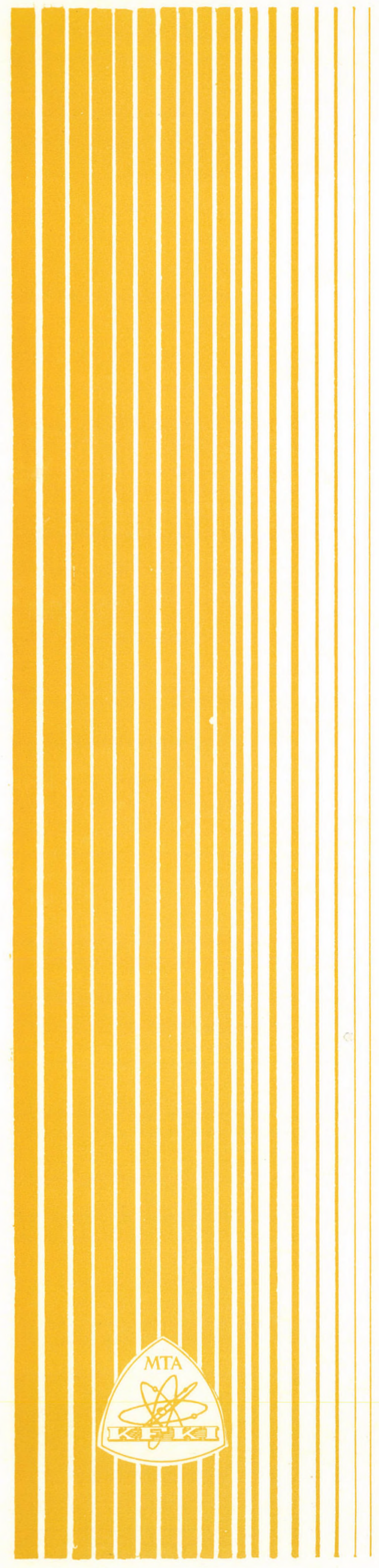

Kiadja a Központi Fizikai Kutató Intézet Felelôs kiadó: Szegõ Károly

Szakmai lektor: Perjés Zoltán

Nyelvi lektor: Hasenfratz Anna

példányszám: 490 Törzsszám: 81-199

Készült a KFKI sokszorositó ủzemében

Felelôs vezetỗ: Nagy Károly

Budapest, 1981. március hó 